\title{
Una visita al Teorema de Stone-von Neumann
}

\section{A visit to the Stone-von Neumann theorem}

\author{
A. GARCIA-CHUNG ${ }^{1}$
}

Recibido: 15 de mayo de 2018/ Aceptado: 3 de junio de 2018

The Stone von-Neumann theorem is a cornerstone in quantum mechanics. It establishes the conditions under which representations of the quantum observables are unitarily equivalent. In this work, we are going to describe, in a rather didactical manner, the main aspects of this theorem and its implications in some current research areas of physics.

El Teorema de Stone-von Neumann constituye una piedra angular de la Mecánica Cuántica. Éste establece las condiciones a partir de las cuales las diferentes representaciones de los observables cuánticos son unitariamente equivalentes. En este trabajo, vamos a describir de una manera didáctica los principales aspectos de este teorema y sus implicaciones en algunas áreas de actualidad en la física.

\section{PALABRAS CLAVES}

Teorema de Stone-von Neumann, Álgebra de Weyl

\section{KEYWORDS}

Stone-von Neumann's theorem, Weyl algebra

PACS

03.65.-w, 03.65.Fd, 03.65.Ca, 04.60.Ds
${ }^{1}$ Departamento de Física, Universidad Autónoma Metropolitana Iztapalapa, San Rafael Atlixco 186, CP 09340, Ciudad de México, México., email: alechung@uam.mx

\section{Agradecimientos}

Le agradezco a Marisol Bermúdez por sus útiles discusiones y realimentaciones durante la preparación de estas notas. También reconozco el apoyo de los subsidios de CONACyT 237351 y 237503.

\section{Acknowledgment}

I thank Marisol Bermúdez for useful discussions and feedback during the preparation of these notes. I also acknowledge the support from CONACyT Grants 237351 and 237503.

\section{I INTRODUCTION}

Muchos científicos percibimos a la mecánica cuántica (MC) como un edificio de ideas bien acabado cuya meta actual es ser implementado y comprendido en un cada vez mayor número de sistemas microscópicos. A más de 100 años de su surgimiento, la estructura fundamental de la MC, sus aspectos conceptuales básicos, permanecen incólumes. La evidencia experimental a favor de esta teoría es abrumadora y si se la combina con la simetría de Lorentz, ésta conduce a la Teoría Cuántica de Campos cuyos resultados experimentales se cuentan entre los más precisos obtenidos por el ser humano (Odom) et al. 2006).

Si pensamos que la columna vertebral de la mecánica cuántica son los postulados de cuantización de Dirac, también conocidos como cuantización canónica (Dirac, 1925 8), entonces esta percepción se vuelve imprecisa. La razón de esto es que todavi'a persisten una serie de limitaciones o problemas abiertos en el programa de cuantización canónica de Dirac (Dirac, 1978). Un ejemplo de esta impresición lo ofrece el Teorema ‘No-Go' de Groenewold, el cual establece que no es posible asociar a cada función 
polinomial sobre el espacio de fase, un único operador abstracto cuya relación de conmutación esté heredada por los corchetes de Poisson del sistema clásico (véase por ejemplo (Hall, 2013) pg. 272 o el excelente artículo de Giulini (Giulini, 2003)). El Teorema 'No-Go' establece con rigor matemático la problemática del ordenamiento de la cuantización canónica el cual es un problema abierto de la MC. Los efectos de este Teorema emergen, por ejemplo, en la cuantización canónica de sistemas altamente no lineales como la gravitación (Isham, 1993, Kuchar, 1993). Claramente, hay aspectos de la MC que constituyen, por así decirlo, grietas en sus fundamentos, y también hay otros que pueden considerarse elementos firmes. En este último caso, destaca el Teorema de Stone-von Neumann del cual tratará este trabajo.

El Teorema de Stone-von Neumann (SvN) (Hall, 2013, Moretti, 2013, Strocchi, 2008) expone los criterios que debe cumplir una representación de las Relaciones de Conmutación Canónicas para que sea unitariamente equivalente a la representación de Schrödinger. Nuestro propósito con el presente trabajo, es hacer un análisis didáctico de este Teorema, para lo cual nos conviene introducir algunos elementos matemáticos claves a modo de motivación. Primeramente, sinteticemos los pasos del programa de cuantización canónica.

El primer paso consiste en realizar la descripción Hamiltoniana ${ }^{1}$ del sistema físico para luego construir el álgebra de Poisson de los observables, denotada como $(\mathbf{F},+, \cdot,\{\})$. Donde $+\mathrm{y} \cdot$ son la suma y la multiplicación escalar habitual en las funciones. La multiplicación entre funciones viene dada no por la composición (que es la llamada multiplicación libre), sino por el corchete de Poisson

$$
\{F, G\}=\frac{\partial F}{\partial x^{a}} \frac{\partial G}{\partial p_{a}}-\frac{\partial F}{\partial p_{a}} \frac{\partial G}{\partial x^{a}} .
$$

En otras palabras, a partir de la descripción Hamiltoniana del sistema físico, dada por $(\Gamma, \Omega, H)$, donde $\Gamma$ es el espacio de fase, $\Omega$ es la 2 -forma simpléctica y $H$ la función Hamiltoniana del sistema, se construye el espacio de las funciones suaves (de preferencia analíticas) sobre $\Gamma$, denotado como $C^{\infty}(\Gamma)$. Estas funciones forman un espacio vectorial con la suma y la multiplicación por escalar " + " y "." dadas anteriormente. A este espacio vectorial se le agrega el corchete de Poisson (multiplicación de álgebra) entre sus elementos usando la 2-forma simpléctica $\Omega$ (ver (Giulini, 2003) para más detalles). De esta manera, obtenemos el álgebra no asociativa $\mathbf{F}$ de los observables del sistema. Nótese que en $\mathbf{F}$ encontramos todas las funciones de interés como el Hamiltoniano $H$, el momento angular $\vec{L}$, etc. El segundo paso consiste en promover cada elemento de $\mathbf{F}$ a un operador abstracto, de tal manera que la relación de conmutación sea compatible con el corchete de Poisson, es decir, si $g_{1}, g_{2}, g_{3} \in \mathbf{F}$, son tales que $\left\{g_{1}, g_{2}\right\}=g_{3}$, entonces al tomar $g_{1} \mapsto \mathbb{G}_{1}, g_{2} \mapsto \mathbb{G}_{2}$ y $g_{3} \mapsto \mathbb{G}_{3}$, debe cumplirse que $\left[\mathbb{G}_{1}, \mathbb{G}_{2}\right]=i \hbar \mathbb{G}_{3}$. Al álgebra de operadores abstractos resultantes la denotamos como $\mathcal{F}$. Este postulado es el que da lugar al problema de ordenamiento mencionado anteriormente en el Teorema 'No-Go' de Groenewold (Giulini, 2003). Como "corolario", debemos mencionar que las condiciones de realidad deben implementarse en este punto, es decir, si definimos la conjugación compleja sobre $\mathbf{F}$, ésta debe extenderse como operación de involución sobre los operadores abstractos. Dicho de otra forma, si el conjugado complejo de $g$ es $h$, es decir, si $\bar{g}=h$, entonces $\mathbb{G}^{*}=\mathbb{H}$, donde $\mathbb{G}^{*}$ es la operación de involución (o "conjugación") sobre los operadores.

El tercer punto en esta versión resumida del formalismo canónico consiste en proporcionar un espacio de Hilbert $\mathcal{H}$, su álgebra de operadores $O(\mathcal{H})$ y una representación de los operadores abstractos de $\mathcal{F}$ en $O(\mathcal{H})$. Conviene recordar que una representación de un álgebra $\mathcal{F}$ es un homomorfismo de álgebras

$$
\pi: \mathcal{F} \rightarrow O(\mathcal{H}) ; \mathbb{G} \mapsto \pi(\mathbb{G})=: \widehat{g},
$$

\footnotetext{
${ }^{1}$ Omitamos por simplicidad el caso de sistemas con constricciones
} 
donde $\pi$ preserva las operaciones del álgebra $\mathcal{F}$ y es comúnmente denotado con el símbolo ${ }^{\wedge}$ sobre la función correspondiente de $\mathbf{F}$.

Podemos ver en este último punto que la elección del espacio de Hilbert debe ser tal que permita la existencia del homomorfismo $\pi$ entre los elementos de $\mathcal{F}$ y los operadores en $O(\mathcal{H})$. Usualmente y como veremos más adelante, se agregan otros requisitos a la representación. Sin embargo, éstos no evitan que exista una infinidad de espacios de Hilbert $\mathcal{H}$ en los que sea posible representar satisfactoriamente el álgebra $\mathcal{F}$. En otras palabras, la elección de $\mathcal{H}$ y con esto, de la representación de $\mathcal{F}$ no es única. Si las representaciones no son únicas entonces existe la posibilidad que den lugar a diferentes resultados físicos, pero sabemos que hasta ahora esto no sucede. Digamos por ejemplo que tenemos otro espacio de Hilbert $\mathcal{H}^{\prime}$ y una representación de $\mathcal{F}$ dada como $\pi^{\prime}$, si $\pi$ es la representación usual de la MC, que llamaremos representación de Schrödinger, ¿qué relación hay entre $\pi$ y $\pi^{\prime}$ ? ¿Qué requisitos debería satisfacer $\pi^{\prime}$ para que ambas representaciones sean equivalentes?

Como veremos en este trabajo, la respuesta a esta pregunta la da el Teorema de SvN. Para comprender este Teorema, se realiza el análisis de las representaciones de $\mathcal{F}$ la cual conduce a varias dificultades matemáticas relacionadas con el dominio de los operadores en $O(\mathcal{H})$. Estas dificultades se evitan considerando un álgebra, introducida por $\mathrm{H}$. Weyl (Weyl, 1950) y que constituye una versión exponencial de las relaciones de conmutación canónicas. Esta álgebra, llamada álgebra de Weyl (Moretti, 2013, Strocchi. 2008), adquiere un carácter 'más' fundamental que la construida usando los conmutadores heredados de los corchetes de Poisson. Esto quiere decir que eventualmente podremos tener una representación del álgebra de Weyl sin que una representación de las relaciones de conmutación, como las conocemos, sea posible. Este tipo de situaciones son comunes en gravitación cuántica por lazos (Thiemann, 2007), en cosmología cuántica por lazos (Agullo and Corichi, 2014, Bojowald, 2008) o en cuantización polimérica (Ashtekar et al. 2003) y llamaremos a este tipo de representaciones representaciones irregulares. En estos casos, los requisitos del Teorema de $\mathrm{SvN}$ no se cumplen y se obtienen representaciones con efectos físicos nuevos y sorprendentes.

Para hacer didáctica la exposición y mostrar el uso del Teorema de $\mathrm{SvN}$, usaremos la representación de Heisenberg (Heisenberg, 1925) y la representación de Schrödinger (Schrödinger, 2003) y mostraremos su equivalencia usando el trabajo de E. Schrödinger (Schrödinger, 2003). Con este propósito, en la Sección II repasaremos los aspectos matemáticos relevantes en la formulación "moderna"de la representación de Heisenberg, también conocida como representación matricial. Luego haremos el tratamiento de la representación de Schrödinger o representación ondulatoria en la Sección III] y haremos la comparación entre ambas en la Sección IV. Aprovecharemos esta sección para introducir representaciones alternativas que motiven aún más la necesidad del Teorema de SvN. Veremos que la comparación de cualquier representación y la representación ondulatoria invita a la construcción del álgebra de Weyl, razón por la cual conviene dedicarle enteramente la Sección $\mathbf{V}$. Por último, en la Sección VI expondremos el Teorema de SvN. Mencionaremos sus consecuencias y discutiremos su alcance, tanto en representaciones irregulares como en la Teoría Cuántica de Campos.

\section{II | HEISENBERG REPRESENTATION}

Comencemos por la formulación matricial la cual, para un sistema con un solo grado de libertad, emplea como ingredientes básicos los operadores abstractos $\nVdash, \mathbb{A}, \mathbb{A}^{*}$. Decimos que son abstractos porque no hemos dado su representación concreta como operadores sobre un espacio de Hilbert. Usamos estos operadores para construir el álgebra de observables, que denotamos como $\mathcal{A}$ y está formada por todas las combinaciones lineales $z \nVdash+\mu \mathbb{A}+v \mathbb{A}^{*}$ siendo $z, \mu, v$ números complejos. Las operaciones de suma, de multiplicación por escalar y de involución, son las naturales como espacio vectorial complejo, es 
decir,

$$
\begin{aligned}
\left(z_{1} \nVdash+\mu_{1} \mathbb{A}+v_{1} \mathbb{A}^{*}\right)+\left(z_{2} \nVdash+\mu_{2} \mathbb{A}+v_{2} \mathbb{A}^{*}\right) & =\left(z_{1}+z_{2}\right) \nVdash+\left(\mu_{1}+\mu_{2}\right) \mathbb{A}+\left(v_{1}+v_{2}\right) \mathbb{A}^{*} \\
\alpha\left(z \nVdash+\mu \mathbb{A}+v \mathbb{A}^{*}\right) & =(\alpha z) \nVdash+(\alpha \mu) \mathbb{A}+(\alpha v) \mathbb{A}^{*}, \\
\left(z \nVdash+\mu \mathbb{A}+v \mathbb{A}^{*}\right)^{*} & =\bar{z} \nVdash+\bar{\mu} \mathbb{A}^{*}+\bar{v} \mathbb{A}
\end{aligned}
$$

con $\alpha \in \mathbb{C}$ y $\bar{z}, \bar{\mu}$ y $\bar{v}$ son los complejos conjugados de $z, \mu$ y $v$ respectivamente. De 5$]$ vemos que $\mathbb{A}^{*}$ es la involución de $\mathbb{A}$, con lo cual $\left(\mathbb{A}^{*}\right)^{*}=\mathbb{A}$. La multiplicación del álgebra está dada por el conmutador de operadores ${ }^{2}$ que toma la forma

$$
\left[z_{1} \nVdash+\mu_{1} \mathbb{A}+v_{1} \mathbb{A}^{*}, z_{2} \nVdash+\mu_{2} \mathbb{A}+v_{2} \mathbb{A}^{*}\right]=\left(\mu_{1} v_{2}-v_{1} \mu_{2}\right) \nVdash,
$$

y que resulta en el ya conocido $\left[\mathbb{A}, \mathbb{A}^{*}\right]=\nVdash$ cuando $z_{1}, z_{2}, v_{1}$ y $\mu_{2}$ son iguales a cero y $\mu_{1}=v_{2}=1$. El álgebra $\mathcal{A}$ construida de esta manera es un álgebra de Lie, lo que implica que satisface la identidad de Jacobi. Esto es fácil de comprobar notando que es además un álgebra nilpotente, es decir, que satisface $\left[z_{1} \nVdash+\mu_{1} \mathbb{A}+v_{1} \mathbb{A}^{*},\left[z_{2} \nVdash+\mu_{2} \mathbb{A}+v_{2} \mathbb{A}^{*}, z_{3} \nVdash+\mu_{3} \mathbb{A}+v_{3} \mathbb{A}^{*}\right]\right]=\left[z_{1} \nVdash+\mu_{1} \mathbb{A}+v_{1} \mathbb{A}^{*},\left(\mu_{2} v_{3}-\mu_{3} v_{2}\right) \nVdash\right]=0$,

de este modo, para cualesquiera elementos $\mathbb{B}_{1}, \mathbb{B}_{2}$ y $\mathbb{B}_{3} \in \mathcal{A}$ tendremos que $\left[\mathbb{B}_{i},\left[\mathbb{B}_{j}, \mathbb{B}_{k}\right]\right]=0$, con $i, j, k=1,2,3$. Usando este resultado obtenemos que la identidad de Jacobi

$$
\left[\mathbb{B}_{1},\left[\mathbb{B}_{2}, \mathbb{B}_{3}\right]\right]+\left[\mathbb{B}_{2},\left[\mathbb{B}_{3}, \mathbb{B}_{1}\right]\right]+\left[\mathbb{B}_{3},\left[\mathbb{B}_{1}, \mathbb{B}_{2}\right]\right]=0,
$$

se satisface ya que cada sumando será nulo. El hecho de que $\mathcal{A}$ sea un álgebra de Lie nos va a ser de mucha utilidad más adelante cuando usemos la fórmula de Baker-Campbell-Hausdorff.

Veamos ahora los elementos importantes del espacio de Hilbert que vamos a emplear para representar al álgebra $\mathcal{A}$. En nuestro caso, tomaremos el espacio de Hilbert de Fock $\mathcal{H}_{\text {Fock }}$ usualmente empleado en los cursos de mecánica cuántica. Los vectores de $\mathcal{H}_{\text {Fock }}$ están formados por combinaciones lineales (posiblemente infinitas) de la forma

$$
|\Psi\rangle=\sum_{j=0} \Psi_{j}|j\rangle, \quad \Psi_{j} \in \mathbb{C},
$$

donde $|j\rangle$ son objetos abstractos que representan una base contable en el espacio de Hilbert de Fock $\mathcal{H}_{\text {Fock. }}$. Los vectores de este espacio tienen norma finita dada como

$$
\||\Psi\rangle \|:=\left(\sum_{j=0}\left|\Psi_{j}\right|^{2}\right)^{1 / 2}<\infty .
$$

Esta norma 10 es la inducida de la expresión $\||\Psi\rangle \|:=\sqrt{\langle\Psi \mid \Psi\rangle}$, donde el producto interno viene dado como

$$
\langle\Psi \mid \Phi\rangle=\left(\sum_{j=0}\langle j| \Psi_{j}^{*}\right)\left(\sum_{s=0} \Phi_{s}|s\rangle\right)=\sum_{j, s=0} \Psi_{j}^{*} \Phi_{s} \delta_{j, s}=\sum_{j=0} \Psi_{j}^{*} \Phi_{j},
$$

y hemos tomado la base $|j\rangle$ como ortonormal: $\langle j \mid s\rangle=\delta_{j, s}$. No es difícil convencerse de que en efecto, la suma de dos vectores de la forma $\sqrt{9}$ es otro vector en $\mathcal{H}_{\text {Fock }}$, es decir, que tiene norma finita.

Pasemos ahora a representar el álgebra $\mathcal{A}$ en $\mathcal{H}_{\text {Fock }}$ dando primeramente la representación de los generadores $\nVdash, \mathbb{A}, \mathbb{A}^{*}$ del álgebra

$$
\nVdash \mapsto \widehat{1} ; \quad \widehat{1}|n\rangle=|n\rangle, \quad \mathbb{A} \mapsto \widehat{A} ; \quad \widehat{A}|n\rangle=\sqrt{n}|n-1\rangle, \quad \mathbb{A}^{*} \mapsto \widehat{A}^{\dagger} ; \quad \widehat{A}^{\dagger}|n\rangle=\sqrt{n+1}|n+1\rangle,
$$

\footnotetext{
${ }^{2}$ Recordemos que el conmutador entre dos operadores $\mathbb{E}$ y $\mathbb{F}$ es la relación $[\mathbb{E}, \mathbb{F}]:=\mathbb{E F}-\mathbb{F E}$.
} 
y verifiquemos que esta representación satisface las relaciones de conmutación dadas en (6) calculando

$$
\left[\widehat{A}, \widehat{A}^{\dagger}\right]|n\rangle=\widehat{A} \widehat{A}^{\dagger}|n\rangle-\widehat{A}^{\dagger} \widehat{A}|n\rangle=(n+1)|n\rangle-n|n\rangle=|n\rangle=\widehat{1}|n\rangle .
$$

Esta representación de los generadores la extendemos a los demás elementos del álgebra $\mathcal{A}$ con lo cual tendremos

$$
a=z \nVdash+\mu \mathbb{A}+v \mathbb{A}^{*} \mapsto \widehat{a}=z \widehat{1}+\mu \widehat{A}+v \widehat{A}^{\dagger} ; \widehat{a}|n\rangle=z|n\rangle+\mu \sqrt{n}|n-1\rangle+v \sqrt{n+1}|n+1\rangle,
$$

e igualmente confirmamos que las relaciones de conmutación (6) se satisfacen

$$
\left[z_{1} \widehat{1}+\mu_{1} \widehat{A}+v_{1} \widehat{A}^{\dagger}, z_{2} \widehat{1}+\mu_{2} \widehat{A}+v_{2} \widehat{A}^{\dagger}\right]|n\rangle=\left(\mu_{1} v_{2}-v_{1} \mu_{2}\right)|n\rangle .
$$

Esta representación es fiel e irreducible como es requerido. Lo primero puede verificarse notando que la identidad del álgebra $\nVdash$ se asocia al operador identidad $\hat{1}$ dado en 12 lo cual permite por ejemplo tener razonablemente bien puesto el problema de autovalores de los operadores. La irreducibilidad se confirma al mostrar que no existen subespacios invariantes de $\mathcal{A}$ en $\mathcal{H}_{\text {Fock }}$ con lo cual toda la 'información relevante' estará contenida en todo el espacio de Hilbert y no en algún subespacio de éste 3 .

Finalmente, notemos que la operación de involución del álgebra $a^{*}$ se asocia a la operación $\widehat{a}^{\dagger}$, es decir, si $a \mapsto \widehat{a}$, entonces $a^{*} \mapsto \widehat{A}^{\dagger}$ y que está última es la operación de adjunto definida como $\left\langle\Psi \widehat{A}^{\dagger} \mid \Phi\right\rangle:=\langle\Psi \mid \widehat{A} \Phi\rangle$. Esto nos permite escribir la acción de los operadores en el dua $]^{4}$ de $\mathcal{H}_{\text {Fock }}$ de la forma

$$
\langle n| \widehat{1}^{\dagger}=\langle n|, \quad\langle n| \widehat{A}^{\dagger}=\sqrt{n}\langle n-1|, \quad\langle n| \widehat{A}=\sqrt{n+1}\langle n+1| .
$$

A la representación del álgebra $\mathcal{A}$ dada en (13) o la dada por la acción de sus generadores (12) la llamamos representación matricial o de Heisenberg y su aspecto matricial se obtiene cuando escribimos las cantidades $\langle m|\widehat{A}| n\rangle$ y $\left\langle m\left|\widehat{A}^{\dagger}\right| n\right\rangle$ en forma de matrices

$$
[\widehat{A}]_{m, n}=\left(\begin{array}{ccccc}
0 & \sqrt{1} & 0 & 0 & \cdots \\
0 & 0 & \sqrt{2} & 0 & \cdots \\
0 & 0 & 0 & \sqrt{3} & \cdots \\
\vdots & \vdots & \vdots & \vdots & \vdots
\end{array}\right), \quad\left[\widehat{A}^{\dagger}\right]_{m, n}=\left(\begin{array}{cccc}
0 & 0 & 0 & \cdots \\
\sqrt{1} & 0 & 0 & \cdots \\
0 & \sqrt{2} & 0 & \cdots \\
0 & 0 & \sqrt{3} & \cdots \\
\vdots & \vdots & \vdots & \vdots
\end{array}\right)
$$

y con esto, cualquier elemento de $\mathcal{A}$ se escribe también en forma matricial identificando $\langle m|\widehat{1}| n\rangle$ con la matriz identidad. Nótese que las matrices resultantes son matrices (cuadradas) de dimensión infinita. Claramente, esta descripción depende notablemente de la base utilizada ( $|n\rangle$ en nuestro caso) y por eso hay que ser cuidadosos con los resultados ya que ello puede implicar que éstos dependan de la base empleada y no del operador en cuestión 5 Por último, cabe mencionar que el trabajo de Heisenberg (Heisenberg, 1925) no empleó las matrices $[\widehat{A}],\left[\widehat{A}^{\dagger}\right]$ sino las correspondientes a los operadores $[\widehat{x}]$ y $[\widehat{p}]$. Sin embargo, el análisis en este trabajo pretende simplificar la exposición usando notación relativamente moderna y compatible con los cursos de Mecánica Cuántica de más actualidad.

El siguiente paso en nuestro análisis consiste en estudiar los dominios de los operadores $\widehat{A}$ y $\widehat{A}^{\dagger}$. Para ello, recordemos que el dominio de un operador, por ejemplo $\widehat{A}$, es el subespacio formado por los

\footnotetext{
3 Tomar representaciones que no sean irreducibles conduce a resultados no físicos p. ej. el espectro del oscilador armónico cuántico resulta no acotado si la representación es reducible. Puede verse Giulini. 2003, para más detalles.

${ }^{4}$ Recordemos que por el Lema de Fréchet-Riesz, el dual $\mathcal{H}^{*}$ de un espacio de Hilbert $\mathcal{H}$ es isomorfo isométrico a éste.

${ }^{5}$ Esta es una de las críticas de J. von Neumann a la formulación de la Mecánica Cuántica hecha por Dirac (ver von Neumann. 1996.).
} 
vectores $|\Psi\rangle \in \mathcal{H}_{\text {Fock }}$ tales que $\widehat{A}|\Psi\rangle \in \mathcal{H}_{\text {Fock }}$. Este subespacio lo denotamos como

$$
\left.\operatorname{Dom}[\widehat{A}]=\left\{|\Psi\rangle \in \mathcal{H}_{\text {Fock }}|\quad \widehat{A}| \Psi\right\rangle \in \mathcal{H}_{\text {Fock }}\right\} .
$$

En otras palabras, dado un vector $|\Psi\rangle \in \mathcal{H}_{\text {Fock }}$ con componentes $\left\{\Psi_{j}\right\}$ dadas como en $[9$, vemos que la acción de $\widehat{A}$ conduce a un nuevo vector $\widehat{A}|\Psi\rangle=\sum_{j=0}^{\infty} \Psi_{j}(\widehat{A}|j\rangle)$ que no está necesariamente en $\mathcal{H}_{\text {Fock }}$. El vector $\widehat{A}|\Psi\rangle$ estará en $\mathcal{H}_{\text {Fock }}$ si sus componentes, que son las cantidades $\left\{\sqrt{j} \Psi_{j}\right\}$, satisfacen la condición de norma finita $\left(\sum_{j=0}\left|\Psi_{j} \sqrt{j}\right|^{2}\right)^{1 / 2}<\infty$. Si el nuevo estado $\widehat{A}|\Psi\rangle$ cumple con esto, entonces decimos que el vector $|\Psi\rangle$ está en el dominio del operador $\widehat{A}$.

Naturalmente, podemos encontrar vectores de $\mathcal{H}_{\text {Fock }}$ que no estén en $\operatorname{Dom}[\widehat{A}]$ como por ejemplo $|\Phi\rangle=\frac{1}{\sqrt{2}}|0\rangle+\sum_{n=1}^{n=\infty} \frac{\sqrt{3}}{\pi^{3} n}|n\rangle$. En este caso, vemos que la norma del vector $\||\Phi\rangle \|$ es una cantidad finita igual a

$$
\||\Phi\rangle||=\left(\frac{1}{2}+\frac{3}{\pi^{6}} \sum_{n=1} \frac{1}{n^{2}}\right)^{1 / 2}=\left(\frac{1}{2}+\frac{3}{\pi^{6}} \frac{\pi^{6}}{6}\right)^{1 / 2}=1,
$$

y por lo tanto pertenece a $\mathcal{H}_{\text {Fock }}$. Sin embargo, la norma del vector $\widehat{A}|\Phi\rangle$ es infinita y por lo tanto no pertenece a $\mathcal{H}_{\text {Fock }}$. Esto lo verificamos haciendo el cálculo explícito de su norma

$$
\| \widehat{A}|\Phi\rangle \|=\frac{\sqrt{3}}{\pi^{3}}\left(1+\frac{1}{2}+\frac{1}{3}+\ldots\right)^{1 / 2}=\frac{\sqrt{3}}{\pi^{3}}(\xi(0))^{1 / 2}
$$

donde $\xi(s)$ es la función Zeta de Riemann la cual diverge en $s=0$. De modo que, $|\Phi\rangle$ es ejemplo de un vector que está en $\mathcal{H}_{\text {Fock }}$ pero que no pertenece al dominio de $\widehat{A}$, es decir, $|\Phi\rangle \notin \operatorname{Dom}[\widehat{A}]$. El dominio del operador $\widehat{A}^{\dagger}$ lo definimos de forma similar

$$
\left.\operatorname{Dom}\left[\widehat{A}^{\dagger}\right]=\left\{|\Psi\rangle \in \mathcal{H}_{\text {Fock }}\left|\quad \widehat{A}^{\dagger}\right| \Psi\right\rangle \in \mathcal{H}_{\text {Fock }}\right\}
$$

y también podemos hacer el ejercicio de encontrar vectores de $\mathcal{H}_{\text {Fock }}$ que no pertenezcan al subespacio $\operatorname{Dom}\left[\widehat{A}^{\dagger}\right]$.

El hecho de que los dominios de los operadores $\widehat{A}$ y $\widehat{A}^{\dagger}$ no cubren todo el espacio de Hilbert $\mathcal{H}_{\text {Fock }}$ indica que éstos son operadores no acotados en $\mathcal{H}_{\text {Fock }}$. Esto se puede entender si recordamos que la norma $\|\widehat{O}\|$ de un operador $\widehat{O}$ sobre un espacio de Hilbert se define como

$$
\|\widehat{O}\|:=\sup _{|\Psi\rangle \in \mathcal{H}_{\text {Fock }}} \frac{\| \widehat{O}|\Psi\rangle \|}{|||\Psi\rangle||}=\sup _{|\Psi\rangle \in \mathcal{H}_{\text {Fock }}} \frac{\left\langle\Psi\left|\widehat{O}^{\dagger} \widehat{O}\right| \Psi\right\rangle^{1 / 2}}{\langle\Psi \mid \Psi\rangle^{1 / 2}}
$$

donde sup indica tomar el supremo del cociente. Si calculamos las normas $\|\widehat{A}\|$ y $\left\|\widehat{A}^{\dagger}\right\|$ vemos que divergen. Para ello basta con tomar estados que no pertenezcan al dominio de los operadores, p. ej., el estado $|\Phi\rangle$ dado anteriormente. De esta manera el numerador en 19 , será divergente y con esto la norma de los operadores $\widehat{A}$ y $\widehat{A}^{\dagger}$.

Por otro lado y antes de continuar, notemos brevemente algunas implicaciones de esta definición de norma, usualmente conocida como norma del supremo o norma uniforme. Primero que el operador $\widehat{1}$ es un operador acotado y con norma $\|\widehat{1}\|=1$ y segundo, que lo mismo sucede con los operadores 
unitarioミ $\S^{6} \widehat{U}$, éstos son acotados y tienen norma igual a 1 , lo cual puede verse fácilmente haciendo el siguiente cálculo

$$
\|\widehat{U}\|=\sup _{|\Psi\rangle \in \mathcal{H}_{F o c k}} \frac{\left\langle\Psi\left|\widehat{U}^{\dagger} \widehat{U}\right| \Psi\right\rangle^{1 / 2}}{\langle\Psi \mid \Psi\rangle^{1 / 2}}=\sup _{|\Psi\rangle \in \mathcal{H}_{\text {Fock }}} \frac{\left\langle\Psi\left|\widehat{U}^{-1} \widehat{U}\right| \Psi\right\rangle^{1 / 2}}{\langle\Psi \mid \Psi\rangle^{1 / 2}}=1 .
$$

Más aún, un análisis de los dominios de estos dos tipos de operadores indica que $\operatorname{Dom}[\widehat{1}]=\mathcal{H}$ y $\operatorname{Dom}[\widehat{U}]=\mathcal{H}$ y aquí omitimos el subíndice "Fock" en $\mathcal{H}$ ya que este resultado es válido para cualquier espacio de Hilbert. Estos resultados nos van a ser útiles en el análisis del álgebra de Weyl en la siguiente sección.

Regresemos entonces a la idea anterior y observemos que si los operadores $\widehat{A}$ y $\widehat{A}^{\dagger}$ son no acotados, entonces las relaciones de conmutación dadas en (14) conducen a la siguiente conexión entre los dominios

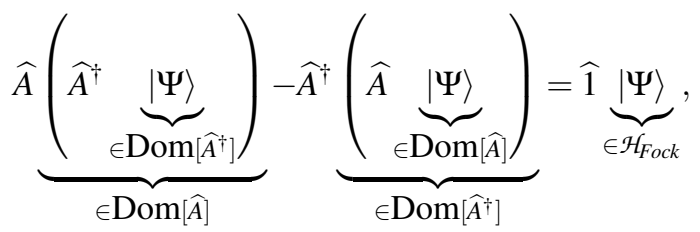

lo cual indica que (14) solo será satisfecha si opera sobre vectores $|\Psi\rangle$ que pertenezcan a los dominios de $\widehat{A}$ y $\widehat{A}^{\dagger}$, y tales que $\widehat{A}^{\dagger}|\Psi\rangle \in \operatorname{Dom}[\widehat{A}]$ y $\widehat{A}|\Psi\rangle \in \operatorname{Dom}\left[\widehat{A}^{\dagger}\right]$. Los vectores que cumplen con estas condiciones forman un subespacio de $\mathcal{H}_{\text {Fock }}$ que denotamos por $\operatorname{Dom}_{\text {Fock }}\left[\left[\widehat{A}, \widehat{A}^{\dagger}\right]\right] \subset \mathcal{H}_{\text {Fock }}$. Es claro que el conjunto de vectores que cumple con estas condiciones forma un subespacio que pertence a la intersección de los dominios de $\widehat{A}$ y $\widehat{A}^{\dagger}$, es decir, $\operatorname{Dom}_{\text {Fock }}\left[\left[\widehat{A}, \widehat{A}^{\dagger}\right]\right] \subset \operatorname{Dom}[\widehat{A}] \cap \operatorname{Dom}\left[\widehat{A}^{\dagger}\right]$. Un ejemplo del tipo de vectores que pertenecen a este subespacio son los formados por series con un número finito de términos.

Estos elementos son los aspectos relevantes de la representación de Heisenberg. Corresponde ahora introducir aquellos necesarios para la descripción de la representación de Schrödinger.

\section{III | SCHRÖDINGER REPRESENTATION}

El álgebra de observables en la mecánica ondulatoria es la formada por los operadores abstractos $\nVdash, \mathbb{X}$ y $\mathbb{P}$. Los elementos de esta álgebra, que denotamos como $\mathcal{S}$, están dados por las combinaciones lineales $z \nVdash+\alpha \mathbb{X}+\beta \mathbb{P}$, donde $z, \alpha, \beta \in \mathbb{C}$. Al igual que $\mathcal{A}$, el álgebra de observables $\mathcal{S}$ es un álgebra de Lie con involución. Las operaciones de multiplicación e involución en $\mathcal{S}$ están dadas de la siguiente forma

$$
\begin{aligned}
{\left[z_{1} \nVdash+\alpha_{1} \mathbb{X}+\beta_{1} \mathbb{P}, z_{2} \nVdash+\alpha_{2} \mathbb{X}+\beta_{2} \mathbb{P}\right] } & =i \hbar\left(\alpha_{1} \beta_{2}-\beta_{1} \alpha_{2}\right) \nVdash, \\
(z \nVdash+\alpha \mathbb{X}+\beta \mathbb{P})^{*} & =\bar{z} \nVdash+\bar{\alpha}+\bar{\beta} \mathbb{P},
\end{aligned}
$$

siendo $\bar{z}, \bar{\alpha}$ y $\bar{\beta}$ los complejos conjugados de $z, \alpha$ y $\beta$ respectivamente. Las relaciones dadas en 22 , son las llamadas Relaciones de Conmutación Canónicas (RCC) y el álgebra de Lie construida con ellas resulta de tomar funciones lineales sobre el espacio de fase del sistema clásico. Si tomamos $z_{1}, z_{2}, \beta_{1} \mathrm{y}$ $\alpha_{2}$ iguales a cero, obtenemos la expresión familiar cuyo único conmutador no nulo es

$$
[\widehat{x}, \widehat{p}]=i \hbar \widehat{1} \text {. }
$$

\footnotetext{
${ }^{6}$ Recordemos que un operador $\widehat{U}$ se dice unitario si $\widehat{U}^{\dagger} \widehat{U}=\widehat{U} \widehat{U}^{\dagger}=\widehat{1}$, siendo $\widehat{U}^{\dagger}$ el adjunto de $\widehat{U}$.
} 
Vemos también de 23 que los operadores abstractos $\mathbb{X}$ y $\mathbb{P}$ son reales ya que cumplen con $\mathbb{X}=\mathbb{X}^{*}$ y $\mathbb{P}=\mathbb{P}^{*}$ a diferencia de los generadores del álgebra $\mathcal{A}$.

Pasemos ahora a analizar el espacio de Hilbert empleado para representar el álgebra $\mathcal{S}$. En nuestro caso, tomaremos el espacio $\mathcal{H}_{S c h}$ formado por las funciones complejas de cuadrado integrable

$$
\mathcal{H}_{S c h}=L^{2}(\mathbb{R}, d x)=\left\{\Psi: \mathbb{R} \rightarrow \mathbb{C} ; x \mapsto \Psi(x), \quad\|\Psi(x)\|:=\left(\int_{-\infty}^{+\infty}|\Psi(x)|^{2} d x\right)^{1 / 2}<\infty\right\},
$$

siendo $\|\Psi(x)\|$ la norma de $\Psi(x)$. Evidentemente, este espacio es bastante grande y contiene funciones muy variadas como por ejemplo, funciones no analíticas o discontinuas, entre otras. Un ejemplo de función de cuadrado integrable que no es continua es el vector $\Phi(x)$ dado como

$$
\Phi(x)=\left\{\begin{array}{cc}
0, & x<1 \\
x^{-1}, & x \geq 1
\end{array}\right.
$$

el cual tiene norma finita

$$
\|\Phi\|=\left(\int_{-\infty}^{+\infty} \bar{\Phi}(x) \Phi(x) d x\right)^{1 / 2}=\left(\int_{1}^{+\infty} \frac{1}{x^{2}} d x\right)^{1 / 2}=\sqrt{1}=1 .
$$

La representación de $\mathcal{S}$ en este espacio es la conocida representación de Schrödinger la cual está dada como

$$
\nVdash \mapsto \widehat{1} ; \quad \widehat{1} \Psi(x)=\Psi(x), \quad \mathbb{X} \mapsto \widehat{x} ; \quad \widehat{x} \Psi(x)=x \Psi(x), \quad \mathbb{P} \mapsto \widehat{p} ; \quad \widehat{p} \Psi(x)=\frac{\hbar}{i} \frac{d}{d x} \Psi(x),
$$

y puede verse con cierto cuidado que 26 es fiel e irreducible y que además, satisface las RCC dadas en (24) o su versión extendida dada en (22)

$$
[\widehat{x}, \widehat{p}] \Psi(x)=\widehat{x}(\widehat{p} \Psi(x))-\widehat{p}(\widehat{x} \Psi(x))=\frac{\hbar}{i} x \frac{d}{d x} \Psi(x)-\frac{\hbar}{i} \frac{d}{d x}(x \Psi(x))=i \hbar \Psi(x) .
$$

Sin embargo, de manera similar a la representación de Heisenberg, los operadores $\widehat{x}$ y $\widehat{p}$ son no acotados. Para ver esto, notemos que los dominios de estos operadores son

$$
\begin{aligned}
\operatorname{Dom}[\widehat{x}] & =\left\{\Psi(x) \in \mathcal{H}_{\text {Sch }} \mid x \Psi(x) \in \mathcal{H}_{\text {Sch }}\right\}, \\
\operatorname{Dom}[\widehat{p}] & =\left\{\Psi(x) \in \mathcal{H}_{\text {Sch }} \mid \frac{\hbar}{i} \frac{d}{d x} \Psi(x) \in \mathcal{H}_{\text {Sch }}\right\},
\end{aligned}
$$

los cuales no abarcan todo el espacio de Hilbert $\mathcal{H}_{S c h}$. Como vimos para el caso de $\mathcal{A}$, esto indicará que las RCC serán satisfechas solo en caso de tomar estados $\Psi(x)$ que pertenezcan al subespacio $\operatorname{Dom}_{S c h}[[\widehat{x}, \widehat{p}]]$ el cual puede deducirse usando un esquema similar al dado en 21)

$$
\underbrace{\widehat{x} \underbrace{\Psi(x)}_{\in \operatorname{Dom}[\hat{p}]})}_{\in \operatorname{Dom}[\hat{x}]}-\widehat{p} \underbrace{(\widehat{x} \underbrace{\Psi(x)}_{\in \operatorname{Dom}[\hat{x}]})}_{\in \operatorname{Dom}[\hat{p}]}=i \hbar \hat{1} \underbrace{\Psi(x)}_{\in \mathcal{H}_{S c h}^{\Psi(x)}}
$$

y de la misma manera tendremos que $\operatorname{Dom}_{S c h}[[\widehat{x}, \widehat{p}]] \subset \operatorname{Dom}[\widehat{x}] \cap \operatorname{Dom}[\widehat{p}]$. Un ejemplo del tipo de vectores que encontraremos en este subespacio son aquellos formados con productos de polinomios con Gaussianas, p. ej., $P^{(n)}(x) e^{-k x^{2}}$, donde $P^{(n)}(x)$ es un polinomio de grado $n$ y $k \in \mathbb{R}$.

Conviene resumir lo hecho hasta aquí, ya que hemos dado los ingredientes principales de ambas 
representaciones, la de Heisenberg y la de Schrödinger. Por un lado tenemos el álgebra $\mathcal{A}$, el espacio de Hilbert $\mathcal{H}_{\text {Fock }}$ y una representación fiel e irreducible de $\mathcal{A}$ en $\mathcal{H}_{\text {Fock }}$ dada en 13 . Los elementos en esta representación, que llamamos de Heisenberg, pueden escribirse en forma de matrices empleando la definición dada en (16) y de aquí sale el nombre de representación matricial. Esto indica que podemos pensar a los operadores $\widehat{a}$ como matrices y a los estados como sucesiones dadas por sus componentes $\left\{\Psi_{j}\right\}$ usando 9 . Mientras que por otro lado, tenemos el álgebra $\mathcal{S}$, el espacio de Hilbert $\mathcal{H}_{S c h}$ y una representación fiel e irreducible de $\mathcal{S}$ en $\mathcal{H}_{S c h}$ dada en 26 . Los operadores en esta representación son operadores diferenciales mientras que los estados son funciones de onda $\Psi(x)$, cuya variable $x$ es una variable continua.

Claramente, ambas representaciones son descripciones cuánticas totalmente diferentes. Esta diferencia se acentúa si tenemos en cuenta que en la exposición que seguimos, no existe ninguna aparente relación entre ellas ambas representaciones. Es decir, no hemos vinculado los elementos de una construcción con los elementos de la otra. En la siguiente subsección mostraremos la relación entre ambas representaciones siguiendo el trabajo de E. Schrödinger. También veremos cómo emergen otras posibles representaciones diferentes de las anteriores dando lugar a una infinidad de representaciones matriciales y ondulatorias.

\section{I COMPARISON AND ALTERNATIVE REPRESENTATIONS}

El primero en mostrar que ambas descripciones eran esencialmente la misma fue E. Schrödinger (Schrödinger, 2003). Su trabajo, notablemente detallado, estableció los requisitos mediante los cuales las matrices obtenidas en la descripción de Heisenberg se relacionaban con las amplitudes obtenidas en la descripción ondulatoria. Mostremos resumidamente las ideas seguidas por E. Schrödinger pero antes vinculemos ambas representaciones.

Relacionar ambas representaciones consiste en dar una transformación entre los espacios de Hilbert donde están definidas que vincule los operadores asociados a cada representación. Para esto, requerimos de una relación biyectiva $f: \mathcal{H}_{\text {Fock }} \rightarrow \mathcal{H}_{\text {Sch }}$ tal que preserve los subespacios

$$
f\left(\operatorname{Dom}_{\text {Fock }}\left[\left[\widehat{A}, \widehat{A}^{\dagger}\right]\right]\right) \subset \operatorname{Dom}_{S c h}[[\widehat{x}, \widehat{p}]], \quad f^{-1}\left(\operatorname{Dom}_{S c h}[[\widehat{x}, \widehat{p}]]\right) \subset \operatorname{Dom}_{F o c k}\left[\left[\widehat{A}, \widehat{A}^{\dagger}\right]\right],
$$

ya que así, vectores que saturan las RCC en un lado, también lo harán en su imagen. Pedimos que $f$ sea además unitaria ya que de este modo, las normas de los estados se preservan de forma exacta

$$
\||\Psi\rangle\|=\| f(|\Psi\rangle)\|, \quad\| \Psi(x)\|=\| f^{-1}(\Psi(x)) \| .
$$

Esta relación entre las amplitudes permite considerar que la acción de los operadores en la representación de Schrödinger, digamos $\widehat{O}_{\mathcal{S}}$, es la imagen de la acción de algún operador en la representación de Fock, digamos $\widehat{O}_{\mathcal{A}}$, es decir,

$$
\widehat{O}_{\mathcal{S}}=f \widehat{O}_{\mathcal{A}} f^{-1}
$$

Ambas acciones se conectan usando la aplicación $f$ y su inversa, de manera que cierra el diagrama de la figura (1).

Sabiendo que $\widehat{x}$ y $\widehat{p}$ satisfacen las RCC 24 , entonces tendremos que deben existir operadores $\widehat{X}_{\mathcal{A}}$ y $\widehat{P}_{\mathcal{A}}$ en $\mathcal{H}_{\text {Fock }}$ tales que satisfagan las mismas RCC, es decir, que se cumple

$$
\begin{aligned}
\widehat{x} \widehat{p}-\widehat{p} \widehat{x} & =\left(f \widehat{X}_{\mathcal{A}} f^{-1}\right)\left(f \widehat{P}_{\mathcal{A}} f^{-1}\right)-\left(f \widehat{P}_{\mathcal{A}} f^{-1}\right)\left(f \widehat{X}_{\mathcal{A}} f^{-1}\right), \\
& =f\left(\widehat{X}_{\mathcal{A}} \widehat{P}_{\mathcal{A}}-\widehat{P}_{\mathcal{A}} \widehat{X}_{\mathcal{A}}\right) f^{-1}=i \hbar \widehat{1}
\end{aligned}
$$




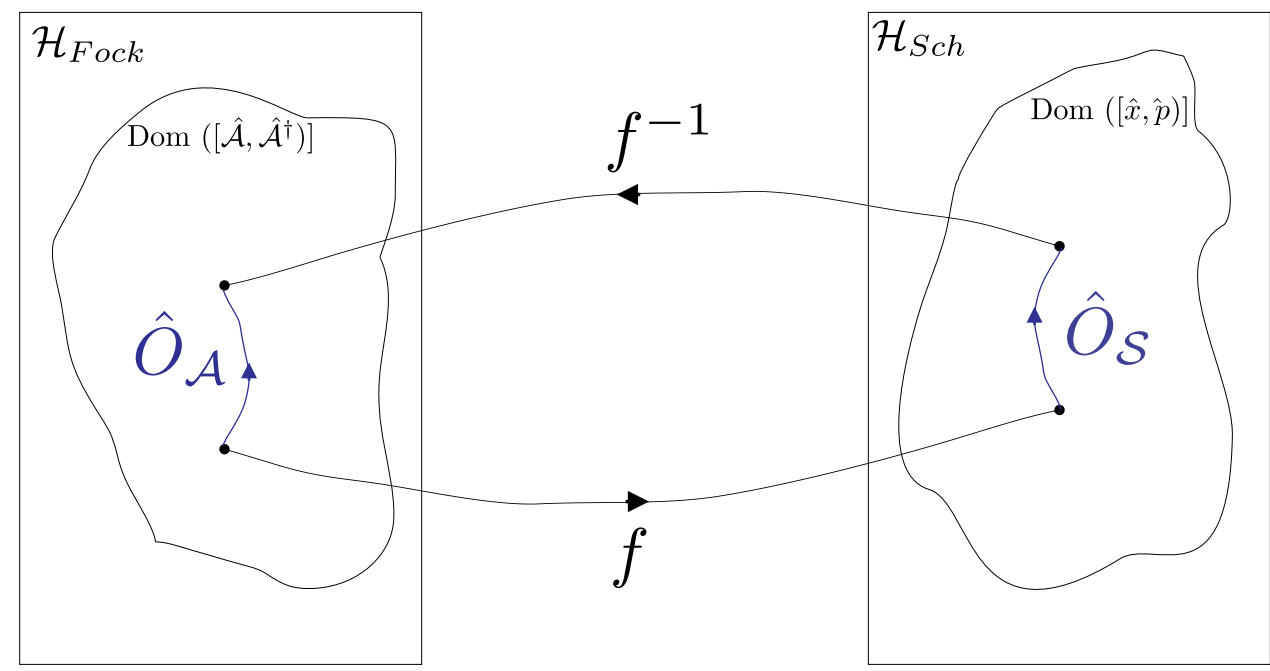

Figura 1: Relación entre los operadores en las representaciones de Heisenberg y Schrödinger.

de lo cual obtenemos

$$
\widehat{X}_{\mathcal{A}} \widehat{P}_{\mathcal{A}}-\widehat{P}_{\mathcal{A}} \widehat{X}_{\mathcal{A}}=i \hbar \widehat{1}_{\mathcal{A}}
$$

donde los operadores $\widehat{X}_{\mathcal{A}}$ y $\widehat{P}_{\mathcal{A}}$ están definidos como

$$
\widehat{X}_{\mathcal{A}}:=f^{-1} \widehat{X}_{\mathcal{S}} f, \quad \widehat{P}_{\mathcal{A}}:=f^{-1} \widehat{X}_{\mathcal{S}} f .
$$

Los operadores $\widehat{X}_{\mathcal{A}}$ y $\widehat{P}_{\mathcal{A}}$ son operadores matriciales ya que actúan sobre $\mathcal{H}_{\text {Fock }}$ y los podemos ver como la versión matricial de los operadores de 'posición' y 'momento' respectivamente. Queremos ahora relacionarlos con los operadores que forman la base del álgebra $\mathcal{A}$ y que esta relación coincida con la dada por W. Heisenberg en (Heisenberg, 1925). Para ello, imponemos que se relacionen con $\widehat{A}$ y $\widehat{A}^{\dagger}$ de la manera más simple, es decir, una relación lineal que además incorpore el hecho de que $\widehat{X}_{\mathcal{A}}$ y $\widehat{P}_{\mathcal{A}}$ son auto-adjuntos en $\mathcal{H}_{\text {Fock }}$. Esto lo hacemos usando una transformación de la forma

$$
\widehat{X}_{\mathcal{A}}=\alpha \widehat{A}+\bar{\alpha} \widehat{A}^{\dagger}, \quad \widehat{P}_{\mathcal{A}}=\beta \widehat{A}+\bar{\beta} \widehat{A}^{\dagger},
$$

siendo $\alpha$ y $\beta$ números complejos que, imponiendo 34 satisfacen la condición

$$
\alpha \bar{\beta}-\bar{\alpha} \beta=i \hbar .
$$

Escribiendo estos números en su forma polar, $\alpha=|\alpha| e^{i \theta_{\alpha}}$ y $\beta=|\beta| e^{i \theta_{\beta}}$, tendremos que la condición anterior toma la siguiente forma

$$
2|\alpha||\beta| \sin \left(\theta_{\alpha}-\theta_{\beta}\right)=\hbar,
$$

la cual establece una condición entre los módulos absolutos $|\alpha|$ y $|\beta|$ y la diferencia entre las fases 
$\Delta \theta:=\theta_{\alpha}-\theta_{\beta}$. Usando esto, los operadores $\widehat{X}_{\mathcal{A}}$ y $\widehat{P}_{\mathcal{A}}$ pasan a escribirse como

$$
\begin{aligned}
\widehat{X}_{\mathcal{A}} & =|\alpha|\left[e^{i\left(\theta_{\beta}+\Delta \theta\right)} \widehat{A}+e^{-i\left(\theta_{\beta}+\Delta \theta\right)} \widehat{A}^{\dagger}\right], \\
\widehat{P}_{\mathcal{A}} & =\frac{\hbar}{2|\alpha| \sin (\Delta \theta)}\left[e^{\left.i \theta_{\beta} \widehat{A}+e^{-i \theta_{\beta}} \widehat{A}^{\dagger}\right] .}\right.
\end{aligned}
$$

Podemos ver de (39) y (40) que al tomar

$$
|\alpha|=\sqrt{\frac{\hbar}{2 m \omega}}, \quad \theta_{\beta}=-\Delta \theta, \quad \Delta \theta=\pi / 2,
$$

recuperamos las relaciones entre los operadores usadas para describir el oscilador armónico cuántico simple, cuyo estudio es primordial en los cursos de Mecánica Cuántica. Estas relaciones son además las mismas usadas por W. Heisenberg en (Heisenberg, 1925).

De esta manera, 35, 39] y 40, constituyen el vínculo entre los operadores de la representación de Fock y la de Schrödinger. Vemos no obstante que existe ambigüedad a la hora de relacionar $\widehat{X}_{\mathcal{A}}, \widehat{P}_{\mathcal{A}}$ con $\widehat{A}$ y $\widehat{A}^{\dagger}$ y que ésta está contenida en los parámetros $|\alpha|$ y $\Delta \theta$. A este punto volveremos más adelante en esta subsección, por ahora continuemos con el análisis de E. Schrödinger.

En su análisis en (Schrödinger, 2003), E. Schrödinger propone la forma explícita de la biyección $f$. Para hacerlo, primero propone que existe una base ortonormal de funciones $\sqrt{\rho(x)} u_{j}(x) \in \mathcal{H}_{S c h}$, con $j=0,1,2, \ldots$ con la cual se construyen las siguientes cantidades

$$
\left\langle u_{j}|\widehat{x}| u_{k}\right\rangle=\int_{-\infty}^{+\infty} d x \rho(x) u_{j}^{*}(x) x u_{k}(x),
$$

siendo $\rho(x)$ la función 'densidad' que es igual a la identidad en nuestro caso. Estas cantidades se hacen coincidir con los elementos de matriz $\left\langle j\left|\widehat{X}_{\mathcal{A}}\right| k\right\rangle$, es decir,

$$
\left\langle j\left|\widehat{X}_{\mathcal{A}}\right| k\right\rangle=\left\langle u_{j}|\widehat{x}| u_{k}\right\rangle,
$$

relacionando así los elementos de la representación de Fock (lado izquierdo) con los de la representación de Schrödinger (lado derecho). Usando este vínculo, vemos que la función $f$ estará dada como

$$
f: \mathcal{H}_{\text {Fock }} \rightarrow \mathcal{H}_{\text {Sch }} ;|j\rangle \mapsto \sqrt{\rho} u_{j}(x),
$$

y puede mostrarse que las RCC se satisfacen de esta manera. El trabajo de Schrödinger no profundiza en el rigor matemático de su construcción y solo establece el vínculo entre ambas construcciones de manera práctica.

Ahora bien, regresemos al asunto de la ambigüedad en los valores de los parámetros $|\alpha|$ y $\Delta \theta$. Cada elección diferente de estos dos parámetros dará lugar a una relación diferente entre $\widehat{x}$ y $\widehat{p}$ con $\widehat{A}$ y $\widehat{A}^{\dagger}$. Si nos ajustamos al esquema seguido por W. Heisenberg en (Heisenberg, 1925), son las RCC dadas por 35) las que tienen carácter fundamental, es decir, los operadores $X_{\mathcal{A}}$ y $P_{\mathcal{A}}$ son la base del álgebra $\mathcal{A}$, mientras que los operadores $\widehat{A}$ y $\widehat{A}^{\dagger}$ son solo operadores auxiliares usados para proveer la representación de $\widehat{X}_{\mathcal{A}}$ y $\widehat{P}_{\mathcal{A}}$. Si nos adherimos a esta idea y tomamos la representación de Heisenberg para $\widehat{X}_{\mathcal{A}}$ y $\widehat{P}_{\mathcal{A}}$ como

$$
\left[\widehat{X}_{\mathcal{A}}\right]_{m, n}=\sqrt{\frac{\hbar}{2 m \omega}}\left(\sqrt{n} \delta_{m, n-1}+\sqrt{n+1} \delta_{m, n+1}\right), \quad\left[\widehat{P}_{\mathcal{A}}\right]_{m, n}=i \sqrt{\frac{\hbar m \omega}{2}}\left(\sqrt{n+1} \delta_{m, n+1}-\sqrt{n} \delta_{m, n-1}\right),
$$

donde los parámetros $|\alpha|, \theta_{\beta}$ y $\Delta \theta$ están dados en 441 , entonces podemos pensar que cada elección de los parámetros en (39) y 40 provee una representación de Heisenberg diferente, etiquetada por $|\alpha|$ y $\Delta \theta$. De esta manera, en lugar de considerar una sola representación matricial, tendremos una infinidad 
de ellas y cada una de las cuales deberá compararse con la de Schrödinger. Esto lo podemos hacer más preciso de la siguiente manera.

Tomemos el espacio de Hilbert de Fock $\mathcal{H}_{\text {Fock }}^{|\alpha|, \Delta \theta}$ con las bases $|j\rangle$, con $j$ un número real cuyo dominio fijaremos más adelante, es decir, no tomaremos necesariamente los enteros no negativos. Para obtener 45 escribimos la representación de $\widehat{X}_{\mathcal{A}}$ y $\widehat{P}_{\mathcal{A}}$ en $\mathcal{H}_{\text {Fock }}^{|\alpha|, \Delta \theta}$ como

$$
\begin{aligned}
\widehat{X}_{\mathcal{A}}|n\rangle & =\sqrt{\frac{\hbar}{2 m \omega}}(\sqrt{n}|n-1\rangle+\sqrt{n+1}|n+1\rangle), \\
\widehat{P}_{\mathcal{A}}|n\rangle & =-i \sqrt{\frac{\hbar m \omega}{2}}(\sqrt{n}|n-1\rangle-\sqrt{n+1}|n+1\rangle) .
\end{aligned}
$$

Si ahora usamos 39$]$ y 40, , obtenemos que las representaciones de $\widehat{A}$ y $\widehat{A}^{\dagger}$ en $\mathcal{H}_{\text {Fock }}^{|\alpha|, \Delta \theta}$ estarán dadas como

$$
\begin{aligned}
\widehat{A}|n\rangle & =\frac{e^{i \varepsilon}}{2|\tilde{\alpha}| \cos \varepsilon}\left[\left(1+|\tilde{\alpha}|^{2} e^{-i \varepsilon} \cos \varepsilon\right) \sqrt{n}|n\rangle+\left(1-|\tilde{\alpha}|^{2} e^{-i \varepsilon} \cos \varepsilon\right) \sqrt{n+1}|n+1\rangle\right], \\
\widehat{A}^{\dagger}|n\rangle & =\frac{e^{-i \varepsilon}}{2|\tilde{\alpha}| \cos \varepsilon}\left[\left(1-|\tilde{\alpha}|^{2} e^{i \varepsilon} \cos \varepsilon\right) \sqrt{n}|n\rangle+\left(1+|\tilde{\alpha}|^{2} e^{i \varepsilon} \cos \varepsilon\right) \sqrt{n+1}|n+1\rangle\right],
\end{aligned}
$$

donde hemos definido $\tilde{\alpha}:=\alpha \sqrt{\frac{2 m \omega}{\hbar}}$ y $\varepsilon:=\Delta \theta-\frac{\pi}{2}$. Puede verse inmediatamente que esta representación, si bien es del tipo matricial, no coincide con la dada en (12) y que cada elección diferente de $\tilde{\alpha}$ y $\varepsilon$ da lugar a una representación diferente de los operadores $\widehat{A}$ y $A^{\dagger}$. Naturalmente, el espacio de Hilbert $\mathcal{H}_{\text {Fock }}^{|\alpha|, \Delta \theta}$ también será diferente. Esto lo podemos observar si calculamos el cuadrado de la norma del estado $\widehat{A}|n\rangle$

$$
\|(\widehat{A}|n\rangle) \|^{2}=\frac{1}{(2|\tilde{\alpha}| \cos \varepsilon)^{2}}\left[2\left(1+|\tilde{\alpha}|^{4} \cos ^{2} \varepsilon\right) n+\left(1-2|\tilde{\alpha}|^{2} \cos ^{2} \varepsilon+|\tilde{\alpha}|^{4} \cos ^{2} \varepsilon\right)\right],
$$

la cual será no negativa si se cumple que $n \geq n_{0}$ donde $n_{0}$ está dado por

$$
n_{0}=-\frac{\left[1-2|\tilde{\alpha}|^{2} \cos ^{2} \varepsilon+|\tilde{\alpha}|^{4} \cos ^{2} \varepsilon\right]}{2\left(1+|\tilde{\alpha}|^{4} \cos ^{2} \varepsilon\right)}
$$

De la representación 48 y 49 para los operadores $\widehat{A}$ y $\widehat{A}^{\dagger}$ vemos que éstos solo agregan cantidades enteras al valor $n$ del estado, es decir, 'trasladan' el valor de $n$ en cantidades $n-1$ ó $n+1$. El valor $n_{0}$ es el mínimo valor posible para tener normas definidas positivas, con lo cual debe cumplirse

$$
\widehat{A}\left|n_{0}\right\rangle=0
$$

con lo cual, es claro que $\left|n_{0}\right\rangle$ es el estado base asociado al espacio de Hilbert $\mathcal{H}_{\text {Fock }}^{|\tilde{\alpha}|, \varepsilon}$, ahora escrito en términos de $\tilde{\alpha}$ y $\varepsilon$. La base en este espacio serán los estados abstractos etiquetados con $n=n_{0}+\mu$, siendo $\mu$ un entero no negativo, es decir, $\left|n_{0}\right\rangle,\left|n_{0}+1\right\rangle,\left|n_{0}+2\right\rangle, \ldots$ De esta forma, hemos obtenido no una, sino infinitas representaciones matriciales y cada una con un estado base $\left|n_{0}\right\rangle$ diferente. Todas ellas tienen una relación análoga a la dada en (44) con la representación de Schrödinger pero, mostrar con rigor la equivalencia unitaria de cada una de ellas con la represetación de Schrödinger no es tarea matemáticamente simple si se tiene en cuenta que los dominios deben preservarse y que los operadores son no acotados en cada una de ellas.

A pesar de lo anterior, es plausible concebir que esta relación unitaria exista y esto conduce al siguiente razonamiento: siendo las representaciones matriciales y ondulatorias construcciones notablemente 
diferentes en su naturaleza, el hecho de que exista una relación unitaria entre ellas sugiere que sus diferencias son más bien un efecto 'accidental' de la representación (de Fock en este caso) y que deben existir características esenciales a partir de las cuales podamos inferir si dada una representación de las RCC ésta es equivalente (unitariamente) a la de Schrödinger.

Esta es justamente la motivación de J. von Neumann como podemos ver de la siguiente cita

[...]Since the systems $F_{Z}$ and $F_{\Omega}$ are isomorphic, and since the theories of quantum mechanics constructed on them are mathematically equivalent, it is to be expected that a unified theory, independent of the accidents of the formal framework selected at the time, and exhibiting only the really essential elements of quantum mechanics, will then be achieved[...]

\section{J. von Neumann, Chapter I, pg 32-33, (von Neumann, 1996)}

En su libro, J. von Neumann se refiere a $F_{\Omega}$ y a $F_{Z}$ a los espacios de funciones construidos con la versión ondulatoria y la matricial respectivamente. Estos espacios contienen las funciones de onda en la versión ondulatoria y la matricial.

Esas características intrínsecas son precisamente las mostradas en el teorema de Stone-von Neumann ${ }^{7}$ La idea es que dada una representación de las RCC, podamos bajo ciertos criterios, dados en el teorema, evaluar si existirá una aplicación $f$ que relaciones la representación dada con la de Schrödinger. Para mostrar los requisitos que debe cumplir la representación pasemos en la siguiente sección a introducir el álgebra de Weyl que será la que nos proporcione.

\section{V | WEYL ALGEBRA}

Vimos en la sección anterior que al trabajar con representaciones de las RCC, los operadores que encontramos resultaban ser operadores no acotados. Como resultado de esto, los dominios de estos operadores no cubrían todo el espacio de Hilbert de la representación. Esto no supone mucho problema en el caso de la representación matricial ya que E. Schrödinger mostró que es equivalente a la representación ondulatoria. No obstante, si tomamos una representación diferente, ni matricial ni ondulatoria, para evaluar su relación con la representación de Schrödinger tendríamos que estudiar los dominios de los operadores fundamentales y luego buscar la biyección $f$ que la vincule con la representación ondulatoria preservando además los dominios. Este proceso puede resultar laborioso y para eludirlo, conviene obtener una representación de las RCC en la que los operadores sean unitarios y de esta manera, los dominios serán todo el espacio de Hilbert ya que los operadores unitarios son acotados. Esto facilitará la posterior comparación con la representación de Schrödinger.

Tomemos como representación de ejemplo la representación de Heisenberg e ignoremos para este fin los resultados de E. Schrödinger. El problema que nos planteamos es entonces construir un operador unitario a partir de los operadores en $\mathcal{A}$. Para ello, ocupamos el Teorema de Stone sobre grupos unitarios mono-paramétricos como viene en (Hall, 2013),

Stone's Theorem: Suppose $U(\cdot)$ is a strongly continuous one-parameter unitary group on $\mathcal{H}$. Then the infinitesimal generator $A$ of $U(\cdot)$ is densely defined and self-adjoint, and $U(t)=e^{i t A}$ for all $t \in \mathbb{R}$. Dicho de otro modo y usando la descripción dada en (Reed and Simon, 1975), este teorema sugiere que si $\widehat{O}$ es un operador auto-adjunto, entonces la serie de potencias $\sum_{n=0}^{\infty}(i s \widehat{O})^{n} / n$ ! cobra sentido y converge al operador $e^{i s \widehat{O}}$ en norma.

\footnotetext{
${ }^{7}$ Se trata en realidad de una serie de teoremas y no un solo teorema como comúnmente pensamos.
} 
Este resultado nos permite entonces elegir operadores auto-adjuntos de $\widehat{O} \in \mathcal{A}$ con los cuales construir operadores unitarios $e^{i s \widehat{O}}$ con $s \in \mathbb{R}$. Sea entonces el operador $z_{1} \widehat{A}+z_{2} \widehat{A}^{\dagger}$ con $z_{1}, z_{2} \in \mathbb{C}$ e impongamos que éste sea auto-adjunto, es decir, $\left(z_{1} \widehat{A}+z_{2} \widehat{A}^{\dagger}\right)^{\dagger}=\left(z_{1} \widehat{A}+z_{2} \widehat{A}^{\dagger}\right)$. Esto impone las siguientes condiciones sobre los números $z_{1}$ y $z_{2}$, a saber, $z_{1}=\bar{z}_{2}$ y con esto el operador $\bar{z} \widehat{A}+z \widehat{A}^{\dagger}$ es auto-adjunto en el espacio de Hilbert $\mathcal{H}_{\text {Fock }}$. Siguiendo el Teorema de Stone, para cada valor $z \in \mathbb{C}$, tendremos un operador unitario en $\mathcal{H}_{\text {Fock }}$ dado como

$$
\widehat{W}(z)=e^{\frac{i}{\sqrt{2}}\left(\bar{z} \widehat{A}+z \widehat{A}^{\dagger}\right)} .
$$

Podemos verificar que es unitario al hacer

$$
\widehat{W}(z)^{\dagger} \widehat{W}(z)=\left(e^{\frac{i}{\sqrt{2}}\left(\bar{z} \widehat{A}+z \widehat{A}^{\dagger}\right)}\right)^{\dagger} e^{\frac{i}{\sqrt{2}}\left(\bar{z} \widehat{A}+z \widehat{A}^{\dagger}\right)}=e^{-\frac{i}{\sqrt{2}}\left(\bar{z} \widehat{A}+z \widehat{A}^{\dagger}\right)} e^{\frac{i}{\sqrt{2}}\left(\bar{z} \widehat{A}+z \widehat{A}^{\dagger}\right)}=\widehat{1}=\widehat{W}(z) \widehat{W}(z)^{\dagger}
$$

y vemos que además, $\widehat{W}(z)^{\dagger}=\widehat{W}(-z)$.

El cálculo explícito de la representación de $\widehat{W}(z)$ en $\mathcal{H}_{\text {Fock }}$ es laborioso, en su lugar, mostremos algunos de los pasos para el cálculo de la amplitud $\langle 0|\widehat{W}(z)| 0\rangle$. Primero vemos que la exponencial se descompone en la suma

$$
\widehat{W}(z)|0\rangle=\sum_{n=0}^{\infty} \frac{(i / \sqrt{2})^{n}\left(\bar{z} \widehat{A}+z \widehat{A}^{\dagger}\right)^{n}}{n !}|0\rangle=|0\rangle+\frac{\frac{i}{\sqrt{2}}\left(\bar{z} \widehat{A}+z \widehat{A}^{\dagger}\right)}{1 !}|0\rangle+\frac{\left(\frac{i}{\sqrt{2}}\right)^{2}\left(\bar{z} \widehat{A}+z \widehat{A}^{\dagger}\right)^{2}}{2 !}|0\rangle+\ldots
$$

y que cada término contribuye dando lugar a una combinación de estados $|0\rangle,|1\rangle,|2\rangle$, etc. Puede verse que solo las potencias pares contienen el estado $|0\rangle$, de modo que solo éstas contribuyen cuando tomemos la primera componente de la matriz asociada a $\widehat{W}(z)$ dada como $\langle 0|\widehat{W}(z)| 0\rangle$. Si tomamos un estado arbitrario $|\Psi\rangle$ que solo tenga componentes en la base de estados pares $|2 j\rangle$, es decir, que se escriba como $|\Psi\rangle=\sum_{j=0}^{j=n} \Psi_{j}|2 j\rangle$, la acción de $\left(\bar{z} \widehat{A}+z \widehat{A}^{\dagger}\right)^{2}$ sobre $|\Psi\rangle$ devuelve un estado que solo tiene componentes en la base par $|2 j\rangle$ pero con coeficientes modificados que dependen de $\Psi_{j}$ linealmente. Las contribuciones de los términos impares $\left(\bar{z} \widehat{A}+z \widehat{A}^{\dagger}\right)^{2 n+1}$ sobre $|\Psi\rangle$ dará lugar a estados que no contienen el estado $|0\rangle$ y por lo tanto se anularán cuando multipliquemos por $\langle 0|$. Es obvio que tomamos estados pares de la forma de $|\Psi\rangle$ ya que $|0\rangle$ y los estados que resulten de la acción de $\left(\bar{z} \widehat{A}+z \widehat{A}^{\dagger}\right)^{2 n}|0\rangle$ tendrán esta forma.

Dicho esto, calculamos la acción del operador $\left(\bar{z} \widehat{A}+z \widehat{A}^{\dagger}\right)^{2}$ sobre $|\Psi\rangle$ quedando como

$$
\begin{aligned}
\left(\bar{z} \widehat{A}+z \widehat{A}^{\dagger}\right)^{2}|\Psi\rangle= & |z|^{2} \Psi_{0}|0\rangle+\bar{z}^{2} \sum_{j=1}^{n} \Psi_{j} \sqrt{(2 j)(2 j-1)}|2 j-2\rangle+|z|^{2} \sum_{j=1}^{n} \Psi_{j}(4 j+1)|2 j\rangle+ \\
& +z^{2} \sum_{j=0}^{n} \Psi_{j} \sqrt{(2 j+1)(2 j+2)}|2 j+2\rangle .
\end{aligned}
$$

Si ahora agrupamos los coeficientes que corresponden a cada elemento de la base, tendremos que la 
forma matricial de $\left(\bar{z} \widehat{A}+z \widehat{A}^{\dagger}\right)^{2}$ se escribe como

$$
\left[\left(\bar{z} \widehat{A}+z \widehat{A}^{\dagger}\right)^{2}\right]=\left(\begin{array}{ccccccc}
|z|^{2} & \sqrt{1 \cdot 2} \bar{z}^{2} & 0 & 0 & 0 & 0 & \cdots \\
\sqrt{1 \cdot 2} z^{2} & 5|z|^{2} & \sqrt{3 \cdot 4} \bar{z}^{2} & 0 & 0 & 0 & \cdots \\
0 & \sqrt{3 \cdot 4} z^{2} & 9|z|^{2} & \sqrt{5 \cdot 6} \bar{z}^{2} & 0 & 0 & \ldots \\
0 & 0 & \sqrt{5 \cdot 6} z^{2} & 13|z|^{2} & \sqrt{7 \cdot 8} \bar{z}^{2} & 0 & \ldots \\
0 & 0 & 0 & \sqrt{7 \cdot 8} z^{2} & 13|z|^{2} & \sqrt{9 \cdot 10} \bar{z}^{2} & \ldots \\
\vdots & \vdots & \vdots & \vdots & \vdots & \vdots & \vdots
\end{array}\right),
$$

y con este resultado, un cálculo laborioso de $\langle 0|\widehat{W}(z)| 0\rangle$ conduce a

$$
\langle 0|\widehat{W}(z)| 0\rangle=1+\left(-\frac{|z|^{2}}{2^{2}}\right)+\frac{1}{2 !}\left(-\frac{|z|^{2}}{2^{2}}\right)^{2}+\cdots+\frac{1}{n !}\left(-\frac{|z|^{2}}{2^{2}}\right)^{n}+\cdots=e^{-\frac{|z|^{2}}{4}}=: \omega_{\text {Fock }}(z) .
$$

Puede verse que la función $\omega_{\text {Fock }}(z)$ es una función continua en la variable $z$. Esta función juega un papel importante en el Teorema de $\mathrm{SvN}$ como veremos más adelante.

Los operadores $\widehat{W}(z)$ tienen otra propiedad más y es la que resulta de considerar su composición sobre estados de $\mathcal{H}_{\text {Fock }}$, es decir, dados dos operadores $\widehat{W}\left(z_{1}\right)$ y $\widehat{W}\left(z_{2}\right)$, podemos definir una multiplicación $\widehat{W}\left(z_{1}\right) \cdot \widehat{W}\left(z_{2}\right)$ que de lugar a otro operador $\widehat{W}\left(z_{3}\right)$ con $z_{3}$ dado por los números complejos $z_{1}$ y $z_{2}$. Para obtener la forma de esta multiplicación nos apoyamos en el hecho de que el álgebra $\mathcal{A}$ es un álgebra de Lie y por lo tanto, la fórmula de Baker-Campbell-Haussdorf (Achilles and Bonfiglioli, 2012, Hall, 2015) puede aplicarse como sigue

$$
\begin{aligned}
\widehat{W}(z) \cdot \widehat{W}\left(z_{2}\right) & =e^{\frac{i}{\sqrt{2}}\left(\bar{z}_{1} \widehat{A}+z_{1} \widehat{A}^{\dagger}\right)} \cdot e^{\frac{i}{\sqrt{2}}\left(\bar{z} \widehat{A}+z \widehat{A}^{\dagger}\right)}=e^{\frac{i}{\sqrt{2}}\left(\bar{z}_{1} \widehat{A}+z_{1} \widehat{A}^{\dagger}\right)+\frac{i}{\sqrt{2}}\left(\bar{z}_{2} \widehat{A}+z_{2} \widehat{A}^{\dagger}\right)-\frac{1}{4}\left[\bar{z}_{1} \widehat{A}+z_{1} \widehat{A}^{\dagger}, \bar{z} 2 \widehat{A}+z_{2} \widehat{A}^{\dagger}\right]} \\
& =e^{-\frac{1}{4}\left(\bar{z}_{1} z_{2}-z_{1} \bar{z}_{2}\right) \widehat{1}+\frac{i}{\sqrt{2}}\left[\overline{\left(z_{1}+z_{2}\right)} \widehat{A}+\left(z_{1}+z_{2}\right) \widehat{A}^{\dagger}\right]}
\end{aligned}
$$

donde hemos considerado que el álgebra $\mathcal{A}$ es nilpotente y por lo tanto la serie en la fórmula de $\mathrm{BCH}$ llega hasta el primer orden. Notemos que en (58) aparece un término proporcional a la identidad con factor $-\frac{1}{4}\left(\bar{z}_{1} z_{2}-z_{1} \bar{z}_{2}\right)$. Debido a que la representación que empleamos es siempre fiel, este término será una fase proporcional al operador identidad con lo cual, la multiplicación (58) se podrá escribir finalmente como

$$
\widehat{W}(z) \cdot \widehat{W}\left(z_{2}\right)=e^{-\frac{1}{4}\left(\bar{z}_{1} z_{2}-z_{1} \bar{z}_{2}\right)} \widehat{W}\left(z_{1}+z_{2}\right),
$$

la cual es conocida como la forma exponencial de las relaciones de conmutación (14). Si escribimos $z_{1}$ y $z_{2}$ en su parte real e imaginaria, $z=x+i y$, tendremos que la multiplicación toma la forma

$$
\widehat{W}\left(x_{1}, y_{1}\right) \cdot \widehat{W}\left(x_{2}, y_{2}\right)=e^{-\frac{i}{2}\left(x_{1} y_{2}-y_{1} x_{2}\right)} \widehat{W}\left(x_{1}+x_{2}, y_{1}+y_{2}\right),
$$

y dos grupos unitarios monoparamétricos, denotados como $\mathcal{U}$ y $\mathcal{V}$, emergen de manera natural cuyos elementos están formados por los operadores $\widehat{U}(x):=\widehat{W}(x, 0)$ y $\widehat{V}(y):=\widehat{W}(0, y)$. Diremos que el grupo $\mathcal{U}$ está formado por los operadores $\widehat{U}(x)$ mientras que el grupo $\mathcal{V}$ lo constituyen los operadores $\widehat{V}(y)$. En ambos, los valores de $x, y \in \mathbb{R}$. Es claro que son grupos de Lie ya que la multiplicación inducida por (60) es cerrada

$$
\begin{aligned}
\widehat{U}\left(x_{1}\right) \cdot \widehat{U}\left(x_{2}\right) & =\widehat{W}\left(x_{1}, 0\right) \cdot \widehat{W}\left(x_{2}, 0\right)=\widehat{W}\left(x_{1}+x_{2}, 0\right)=\widehat{U}\left(x_{1}+x_{2}\right), \\
\widehat{V}\left(y_{1}\right) \cdot \widehat{V}\left(y_{2}\right) & =\widehat{W}\left(0, y_{1}\right) \cdot \widehat{W}\left(0, y_{2}\right)=\widehat{W}\left(0, y_{1}+y_{2}\right)=\widehat{V}\left(y_{1}+y_{2}\right),
\end{aligned}
$$

mientras que, vistos como operadores, la multiplicación entre los elementos de un grupo con los del 
otro queda como

$$
\widehat{U}(x) \widehat{V}(y)=e^{-i x y} \widehat{V}(y) \widehat{U}(x) .
$$

Notemos que estos grupos no son una simple coincidencia de la elección en los valores de $z$ ya que si escribimos la relación en la forma de los generadores veremos

$$
\begin{aligned}
& \widehat{U}(x)=e^{\frac{i}{\sqrt{2}}\left(x \widehat{A}+x \widehat{A}^{\dagger}\right)}=e^{\frac{i}{\sqrt{2}} x\left(\widehat{A}+\widehat{A}^{\dagger}\right)}=e^{i x \sqrt{\frac{m \omega}{\hbar}} \widehat{X}_{\mathcal{A}}}, \\
& \widehat{V}(y)=e^{\frac{i}{\sqrt{2}}\left(-i y \widehat{A}+i y \widehat{A}^{\dagger}\right)}=e^{\frac{y}{\sqrt{2}}\left(\widehat{A}-\widehat{A}^{\dagger}\right)}=e^{i y \sqrt{\frac{1}{\hbar m \omega}} \widehat{P}_{\mathcal{A}}},
\end{aligned}
$$

donde hemos usado (39) y (40) con los valores de la relación de Heisenberg para los parámetros dada en 41 . Vemos entonces que los grupos $\mathcal{U}$ y $\mathcal{V}$ corresponden a la acción de los operadores unitarios generados con $\widehat{X}_{\mathcal{A}}$ y $\widehat{P}_{\mathcal{A}}$ respectivamente. Este resultado es notable por la siguiente razón: si empezamos nuestro análisis con la representación de alguna álgebra $\mathcal{A}^{\prime}$ y sin hacer referencia a cuáles serían los operadores $\widehat{X}_{\mathcal{A}^{\prime}}$ y $\widehat{P}_{\mathcal{A}^{\prime}}$, entonces la construcción de los operadores $\widehat{W}(z)$ con su multiplicación correspondiente 60 y la posterior dedución de los grupos con $\mathcal{U}$ y $\mathcal{V}$, ofrece una representación exponencial de los que 'deberían' ser los operadores $\widehat{X}_{\mathcal{A}^{\prime}}$ y $\widehat{P}_{\mathcal{A}^{\prime}}$ para el álgebra $\mathcal{A}^{\prime}$. Así, conocer una representación de $\mathcal{U}$ y $\mathcal{V}$ permite 'deducir' los operadores que saturan las RCC. Las ventajas de este análisis son que debido a que los $\widehat{W}(z)$ son unitarios, y en consecuencia también los $\widehat{U}(x)$ y $\widehat{V}(y)$, todo el estudio se realizará en el espacio de Hilbert completo sin tener que prestar atención al asunto de los dominios. Es decir, si pensamos a cualesquiera de las multiplicaciones [59, o 60, o la dada por la terna 61, 62) y 63, , como las versiones exponenciales de las RCC, éstas permitirán caracterizar y estudiar las representaciones correspondientes en todo el espacio de Hilbert.

Por otro lado, consideremos ahora las siguientes combinaciones lineales finitas

$$
\widehat{w}=\sum_{j=1}^{n} c_{j} \widehat{W}\left(z_{j}\right),
$$

las cuales permiten considerar a los operadores $\widehat{W}(z)$ como los generadores de una base de un espacio vectorial complejo siendo $c_{j} \in \mathbb{C}$. Nótese que $\widehat{w}$ sigue siendo un operador matricial sobre $\mathcal{H}_{\text {Fock }}$. Este espacio vectorial de operadores matriciales tiene además la multiplicación (59), por lo cual es además un álgebra a la que además le podemos agregar la operación de involución dada como

$$
*(\widehat{w})=\widehat{w}^{*}=\sum_{j=1}^{n} \bar{c}_{j} \widehat{W}\left(-z_{j}\right),
$$

y una norma del supremo igual a la dada en la sección anterior

$$
\|\widehat{w}\|=\sup _{|\Psi\rangle} \frac{\| \widehat{w}|\Psi\rangle \|}{\|(|\Psi\rangle) \|} .
$$

Vemos entonces que $\|\widehat{W}(z)\|=1$ y por lo tanto, todos los operadores $\widehat{w}$ serán acotados. Esto hace que el álgebra obtenida sea un álgebra normada que además, no contiene los puntos límites formados con sucesiones $\left\{\widehat{w}_{n}\right\}$. Si completamos el álgebra con estos puntos límites, es decir, agregar aquellos elementos $\left\{\widehat{w}_{n}\right\}$ tales que los límites $\left\|\widehat{w}_{n}\right\|$ existen, entonces al álgebra resultante la llamamos álgebra de Weyl y la denotamos como $\mathcal{W}$.

La relevancia de $\mathcal{W}$ es que desde el punto de vista matemático, es el álgebra asociativa más 'chica' que 'contiene' al álgebra $\mathcal{A}$. Esto quiere decir que es la envoltura universal de $\mathcal{A}$ y que muchas de sus propiedades se pueden estudiar sin aludir a una representación concreta. Este hecho, unido al resultado anterior de que permite deducir los grupos $\mathcal{U}$ y $\mathcal{V}$ con los cuales recuperar las RCC sugiere 
que podemos considerar al álgebra $\mathcal{W}$ como la estructura matemática fundamental y no al álgebra de observables $\mathcal{A}$. En términos de J. von Neumann, es esta álgebra la que contiene la información relacionada con las características intrínsecas a estudiar de las RCC. De hecho, este es el enfoque de cuantizaciones no usuales como aquellas usadas en loop quantum gravity, loop quantum cosmology y polymer quantum mechanics donde se tienen representaciones de $\mathcal{W}$ pero no de $\mathcal{A}$ o de $\mathcal{S}$. Algo de esto comentaremos al final de este trabajo.

Corresponde ahora realizar una construcción similar de $\mathcal{W}$ pero usando el álgebra de Schrödinger $\mathcal{S}$. Siguiendo los pasos anteriores, tomamos los operadores

$$
\begin{aligned}
& \widehat{u}(\mu):=e^{i \frac{\mu \widehat{x}}{\hbar}} \rightarrow \widehat{u}(\mu) \Psi(x)=e^{i \frac{\mu \widehat{\widehat{x}}}{\hbar}} \Psi(x)=e^{i \frac{\mu x}{\hbar}} \Psi(x), \\
& \widehat{v}(v):=e^{i \frac{v \widehat{p}}{\hbar}} \rightarrow \widehat{v}(v) \Psi(x)=e^{i \frac{v \widehat{p}}{\hbar}} \Psi(x)=\Psi(x+v),
\end{aligned}
$$

y con esto al aplicar el operador $\widehat{v}(v)$ en 69 y $\widehat{u}(\mu)$ en $(70)$, deducimos la multiplicación de los operadores $\widehat{u}(\mu)$ y $\widehat{v}(\mathrm{v})$, la que tomará la forma

$$
\widehat{u}(\mu) \widehat{v}(v)=e^{-\frac{i \mu \nu}{\hbar}} \widehat{v}(v) \widehat{u}(\mu) .
$$

Desde aquí podemos ver la similitud que guarda la relación (71) con la multiplicación dada en (63). Para hacerla más general, definamos ahora la exponencial de los operadores $\mu \widehat{x}+v \widehat{p}$, en analogía a la que usamos para construir $\widehat{W}(z)$, pero en este caso, tendremos un nuevo operador $\widehat{W}^{\prime}(\mu, v)$ dado como

$$
\widehat{W}^{\prime}(\mu, v)=e^{\frac{i}{\hbar}(\mu \widehat{x}+v \widehat{p})}
$$

el cual será unitario al tomar $\mu, v \in \mathbb{R}$ y cuya multiplicación toma la forma

$$
\begin{aligned}
\widehat{W}^{\prime}\left(\mu_{1}, v_{1}\right) \cdot \widehat{W}^{\prime}\left(\mu_{2}, v_{2}\right) & =e^{\frac{i}{\hbar}\left(\mu_{1} \widehat{x}+v_{1} \widehat{p}\right)} \cdot e^{\frac{i}{\hbar}\left(\mu_{2} \widehat{x}+v_{2} \widehat{p}\right)}=e^{-\frac{i}{2 \hbar}\left(\mu_{1} v_{2}-v_{1} \mu_{2}\right)+\frac{i}{\hbar}\left[\left(\mu_{1}+\mu_{2}\right) \widehat{x}+\left(v_{1}+v_{2}\right) \widehat{p}\right]}, \\
& =e^{-\frac{i}{2 \hbar}\left(\mu_{1} v_{2}-v_{1} \mu_{2}\right)} \widehat{W}^{\prime}\left(\mu_{1}+\mu_{2}, v_{1}+v_{2}\right) .
\end{aligned}
$$

$\mathrm{Al}$ álgebra construida con estos generadores la denotamos como $\mathcal{W}^{\prime}$ y al igual que $\mathcal{W}$, será el completamiento en la norma del supremo de las combinaciones lineales finitas generadas con $\widehat{W}^{\prime}(\mu, v)$. El álgebra $\mathcal{W}^{\prime}$ es la envoltura universal del álgebra $\mathcal{S}$, es decir, es el álgebra asociativa más chica que contiene las RCC 24] y sus elementos serán las combinaciones lineales

$$
\widehat{w}^{\prime}=\sum_{j} c_{j} \widehat{W}^{\prime}\left(\mu_{j}, v_{j}\right)
$$

posiblemente infinitas tales que $\left\|\widehat{w}_{n}^{\prime}\right\|$ converja siendo $\left\{\widehat{w}_{n}^{\prime}\right\}$ los elementos de una sucesión del álgebra. Dicho en otras palabras, $\mathcal{W}^{\prime}$ es el álgebra de Weyl asociada a $S$.

Vemos que de forma análoga al álgebra $\mathcal{W}$, los generadores $\widehat{W}^{\prime}(\mu, v)$ se pueden relacionar con los operadores unitarios $\widehat{U}(\mu)$ y $\widehat{V}(v)$ de la forma $\widehat{U}(\mu)=\widehat{W}^{\prime}(\mu, 0)$ y $\widehat{V}(v)=\widehat{W}^{\prime}(0, v)$. En este caso, tendremos que

$$
\widehat{W}^{\prime}(\mu, v)=e^{\frac{i}{2 \hbar} \mu \nu} \widehat{u}(\mu) \widehat{v}(v),
$$

y usando este resultado, podemos calcular la representación de $\mathcal{W}^{\prime}$ en $\mathcal{H}_{S c h}$ la cual resulta estar dada como

$$
\widehat{W}^{\prime}(\mu, v) \Psi(x)=e^{\frac{i}{2 \hbar} \mu v} e^{\frac{i}{\hbar} \mu x} \Psi(x+v),
$$

para todo $\Psi(x) \in \mathcal{H}_{S c h}$. Si ahora consideramos la amplitud $\left\langle\Psi\left|\widehat{W}^{\prime}(\mu, v)\right| \Psi\right\rangle$ esta puede calcularse 
inmediatamente de la forma

$$
\left\langle\Psi\left|\widehat{W}^{\prime}(\mu, v)\right| \Psi\right\rangle=\int_{-\infty}^{+\infty} d x \Psi^{*}(x)\left(\widehat{W}^{\prime}(\mu, v) \Psi(x)\right)=\int_{-\infty}^{+\infty} d x \Psi^{*}(x)\left(e^{\frac{i}{2 \hbar} \mu v} e^{\frac{i}{\hbar} \mu x} \Psi(x+v)\right),
$$

con lo cual, para ilustrar un ejemplo de este cálculo, tomamos el estado normalizado $\Psi_{0}(x):=$ $\left(\frac{2 \Lambda}{\pi}\right)^{1 / 4} e^{-\Lambda x^{2}}$ y vemos que la amplitud de $\widehat{W}^{\prime}(\mu, v)$ es de la forma

$$
\left\langle\Psi_{0}\left|\widehat{W}^{\prime}(\mu, v)\right| \Psi_{0}\right\rangle=e^{-\frac{1}{2}\left(\Lambda v^{2}+\frac{\mu^{2}}{4 \Lambda \hbar^{2}}\right)}=: \omega_{S c h r}(\mu, v)
$$

donde, una vez más, la función $\omega_{S c h r}(\mu, v)$ resulta ser una función continua en las variables $\mu$ y $v$.

Tenemos entonces dos álgebras de Weyl, cada una construida con un álgebra de Lie diferente. El álgebra $\mathcal{W}$ se obtuvo con los elementos de $\mathcal{A}$ representados en $\mathcal{H}_{\text {Fock }}$ mientras que el álgebra $\mathcal{W}^{\prime}$ se obtuvo con la representación de los operadores de $\mathcal{S}$ en $\mathcal{H}_{S c h}$. Si analizamos la multiplicación 73 vemos que también guarda cierta similitud con la dada en (59). De hecho, podemos definir un isomorfismo entre las álgebras de Weyl $\mathcal{W}$ y $\mathcal{W}^{\prime}$

$$
\alpha: \mathcal{W} \rightarrow \mathcal{W}^{\prime} ; \widehat{W}(x, y) \mapsto \widehat{W}^{\prime}(\mu, v)=\alpha(\widehat{W}(x, y)),
$$

de tal manera que $\alpha$ preserve las operaciones de multiplicación del álgebra y las de espacio vectorial complejo y es además biyectiva. Para que la multiplicación se preserve, podemos tomar $\alpha$ de tal forma que relacione los parámetros de los generadores, es decir

$$
\alpha(\widehat{W}(x, y))=\widehat{W}^{\prime}(\tilde{\alpha}(x, y)), \quad(\mu, v):=\tilde{\alpha}(x, y), \quad \rightarrow \quad\left(\begin{array}{c}
\mu \\
v
\end{array}\right):=\left(\begin{array}{ll}
\alpha_{11} & \alpha_{12} \\
\alpha_{21} & \alpha_{22}
\end{array}\right)\left(\begin{array}{l}
x \\
y
\end{array}\right)
$$

en la cual $\tilde{\alpha}$ es una matriz que cumple con la siguiente condición

$$
\left(\begin{array}{ll}
\alpha_{11} & \alpha_{12} \\
\alpha_{21} & \alpha_{22}
\end{array}\right)^{T}\left(\begin{array}{cc}
0 & 1 \\
-1 & 0
\end{array}\right)\left(\begin{array}{ll}
\alpha_{11} & \alpha_{12} \\
\alpha_{21} & \alpha_{22}
\end{array}\right)=\left(\begin{array}{cc}
0 & 1 \\
-1 & 0
\end{array}\right)
$$

es decir, la relación entre los parámetros es una relación canónica! Esta relación conduce en el caso de sistemas de un grado de libertad una única condición dada como

$$
\alpha_{11} \alpha_{22}-\alpha_{12} \alpha_{21}=1
$$

con cual apreciamos que existen en realidad una infinidad de isomorfismos entre las álgebras de Weyl $\mathcal{W}$ y $\mathcal{W}^{\prime}$. Esto implica que las álgebras $\mathcal{W}$ y $\mathcal{W}^{\prime}$ son esencialmente la misma álgebra ya que existen infinitas formas de relacionarlas isomórficamente. En otras palabras: el álgebra de Weyl asociada a un álgebra de Lie dada es única módulo isomorfismos.

Por otro lado, la Teoría de Álgebras de Lie proporciona el Teorema de Poincaré-Birkhoff-Witt (PBW) (Dixmier, 1977) con el cual podemos establecer que la envoltura universal de cualquier álgebra de Lie es única módulo isomorfismos. Recordemos que el álgebra de Weyl asociada a un álgebra de Lie es lo que llamamos su envoltura universal. De modo que un efecto del Teorema de PBW, es que si dos álgebras de Lie son isomorfas, entonces sus envolturas universales serán igualmente isomorfas, o lo que es lo mismo, ambas tendrán la misma envoltura universal módulo isomorfismos. Esto es lo que sucede en nuestro caso, las álgebras de Lie $\mathcal{A}$ y $\mathcal{S}$ son isomorfas y por lo tanto, sus envolturas también serán isomorfas.

Ahora bien, el alcance de la construcción del álgebra de Weyl es que ésta es única módulo isomorfismos y que es además un álgebra asociativa que contiene las Relaciones de Canónicas de Conmutación. La 
ventaja de la unicidad es evidente, solo hay un álgebra de Weyl, y el que sea asociativa nos permite hacer uso de las poderosas herramientas del análisis funcional como por ejemplo la construcción GNS (Haag, 2012, Strocchi, 2008). Otra ventaja es la que habíamos mencionado anteriormente y tiene que ver con la existencia de los grupos mono-paramétricos unitarios los cuales actúan sobre todo el espacio de Hilbert y están formados por operadores acotados.

Pasemos entonces a la siguiente sección donde expondremos las ideas relacionadas con el Teorema de Stone-von Neumann.

\section{VI | STONE-VON NEUMANN THEOREM}

En las secciones anteriores hemos procurado enfatizar varios aspectos relevantes que son usualmente omitidos en los cursos de mecánica cuántica. A saber, que las RCC admiten no una (la de Schrödinger) sino infinitas posibles representaciones, todas fieles e irreducibles. Como ejemplo de esto, mostramos la cuantización matricial introducida inicialmente por W. Heisenberg y concluimos que también existían no una, sino infinitas representaciones matriciales etiquetadas en los parámetros $|\tilde{\alpha}|$ y $\Delta \theta$ con los cuales relacionábamos $\mathcal{A}$ con $\mathcal{S}$. Claramente, no son éstas las únicas representaciones posibles de las RCC, véase por ejemplo la representación de $\mathcal{A}$ usando funciones holomorfas conocida como cuantización de Segal-Bargmann (Bargmann, 1961; Hall, 2013).

Vimos entonces la propuesta de E. Schrödinger de relacionar la cuantización matricial con la ondulatoria vinculando los operadores de la representación de $\mathcal{A}$ con aquellos de la representación de $\mathcal{S}$ y construyendo la aplicación $f$ entre los espacios de Hilbert $\mathcal{H}_{\text {Fock }}$ y $\mathcal{H}_{S c h}$. Este hecho muestra que, aún cuando dos representaciones sean notablemente diferentes, estas pueden ser unitariamente equivalentes y tener así el mismo contenido físico. Esto conduce a plantearse cuáles son las características intrínsecas que debemos considerar de una representación tales que nos proporcionen la información de si la representación en cuestión es equivalente o no a la de Schrödinger. Claramente, el ejercicio realizado por E. Schrödinger puede ser laborioso si pretendemos llevarlo a cabo para cada una de las posibles representaciones. Esto conduce a la idea de J. von Neumann de estudiar las características intrínsecas en lugar de definir $f$. Estas características nos dirán esencialmente si existe alguna función $f$ o no.

Los dominios son un obstáculo para estudiar la relación entre las representaciones y por eso se construyen operadores unitarios ya que éstos actúan sobre todo el espacio de Hilbert. Con esto en mente, se definen los operadores $\widehat{W}(z)$ y $\widehat{W}^{\prime}(\mu, v)$ con los cuales se construyen las álgebras de Weyl $\mathcal{W}$ y $\mathcal{W}^{\prime}$ respectivamente. Luego vimos que ambas son isomorfas y luego establecimos un criterio de unicidad (módulo isomorfismos) para el álgebra de Weyl.

El hecho de que $\mathcal{W}$ es asociativa, sus generadores son operadores unitarios y es además única, nos permite considerarla como el álgebra que tiene en sí las características intrínsecas de las RCC que estamos buscando. Esto quiere decir que dada un álgebra de Lie cualquiera, digamos $\mathcal{B}$ para estudiar su relación con las RCC dadas en la representación de Schrödinger, debemos construir su 'versión' exponencial, es decir, el álgebra de Weyl asociada a ésta, $\mathcal{W}_{\mathcal{B}}$. Luego vemos si cumple los requisitos del Teorema de Stone-von Neumann y si es afirmativo, entonces existe una función $f$ entre las representación de $\mathcal{B}$ y $\mathcal{S}$.

Stone-von Neumann's Theorem: Todas las representaciones débilmente continuas e irreducibles del álgebra de Weyl son unitariamente equivalentes (Hall, 2013, Moretti, 2013; Strocchi, 2008).

Vemos entonces que el requisito que deben satisfacer las representaciones, es que éstas sean débilmente continuas e irreducibles. Todas las representaciones con las que hemos trabajado y que usaremos son irreducibles, de modo que solo resta por comprender el criterio de continuidad débil.

Regresemos al caso del álgebra hipotética $\mathcal{B}$ y su versión à la Weyl, $\mathcal{W}_{\mathcal{B}}$. Tomemos un elemento 
arbitrario $\widehat{a} \in \mathcal{W}_{\mathcal{B}}$. Este operador será una combinación lineal arbitraria $\widehat{a}=\sum_{n} c_{n} \widehat{W}_{\mathcal{B}}\left(L_{n}\right)$, donde $L_{n}$ son las etiquetas del generador de Weyl $\widehat{W}_{\mathcal{B}}\left(L_{n}\right)$. Tomemos por otro lado un vector $|\Psi\rangle$ cualquier del espacio de Hilbert $\mathcal{H}_{\mathcal{B}}$, en el que tenemos la representación de $\mathcal{B}$ y calculemos la amplitud $\langle\Psi|\widehat{a}| \Psi\rangle=: \omega_{\mathcal{B}}\left(\left\{L_{n}\right\}\right)$, la cual resultará ser una función de los parámetros $L_{n}$. Si la función $\omega_{\mathcal{B}}\left(\left\{L_{n}\right\}\right)$ es una función continua, entonces decimos que la representación de $\mathcal{W}_{\mathcal{B}}$ es una representación débilmente continua.

Veamos la representación de Schrödinger y tomemos $\widehat{a}=\widehat{W}^{\prime}(\mu, v)$ y $|\Psi\rangle=\Psi_{0}(x)$. Vemos entonces que $\omega_{S c h r}(\mu, v)$ dada en $(78)$ es una función continua en los parámetros $\mu$ y $v$. Podemos hacer este cálculo con cualquier elemento de $\mathcal{W}^{\prime}$ y con cualquier vector de $\mathcal{H}_{S c h}$ y el resultado será el mismo: la función $\omega_{S c h r}$ es siempre una función continua. Esto quiere decir que la representación de Schrödinger cumple con los requisitos establecidos en el Teorema de SvN.

En el caso de la representación de Heisenberg, vemos que el análisis es muy similar. Por ejemplo, tomemos $|\Psi\rangle=|0\rangle$ y $\widehat{a}=\widehat{W}(z)$. En este caso, la función $\omega_{\text {Fock }}(z)$ viene dada en 57 y es como ya vimos, una función continua en $z$. De modo que es otra representación que también cumple con los requisitos establecidos por el Teorema de SvN. Es claro entonces que el Teorema de SvN indica que la representación de Heisenberg y la representación de Schödinger son unitariamente equivalentes, algo que ya sabíamos gracias al trabajo de E. Schrödinger pero que hemos obtenido haciendo uso del Teorema de SvN.

El alcance de este Teorema es notable si tenemos en cuenta que cualquier otra representación que cumpla con los requisitos del Teorema será unitariamente equivalente a la representación ondulatoria y a la representación matricial. Es decir, como todas las representaciones empleadas en la descripción convencional de la MC cumplen con los requisitos del Teorema, TODAS son unitariamente equivalentes a la representación de Schrödinger o lo que es lo mismo, la representación de Schrödinger es la única representación débilmente continua e irreducible módulo transformaciones unitarias. Es por esto que al cuantizar canónicamente un sistema, no nos preocupamos en general si la representación es o no equivalente a la de Schrödinger.

Veamos ahora un contraejemplo y consideremos el álgebra de Weyl dada como $\mathcal{W}^{\prime}$. Sea entonces un espacio de Hilbert dado como $\mathcal{H}^{\prime}=L^{2}\left(\mathbb{R}_{d}, d x_{c}\right)$, donde $\mathbb{R}_{d}$ es la linea real con topología discreta y $d x_{c}$ es la medida contable sobre $\mathbb{R}_{d}$. Los vectores en este espacio son las combinaciones de la forma

$$
\Psi(x)=\sum_{\left\{x_{j}\right\}} \Psi_{x_{j}} \delta_{x_{j}, x},
$$

donde $\left\{x_{j}\right\}$ es un conjunto arbitrario de puntos de $\mathbb{R}_{d}, \Psi_{j} \in \mathbb{C}$ y $\delta_{x_{j}, x}$ es la delta de Kronecker. Este espacio de Hilbert es no separable, lo cual puede verse al notar que los elementos de la base son precisamente las delta de Kronecker $\delta_{x_{j}, x}$ y que éstas forman un conjunto no contable. Los coeficientes $\Psi_{x_{j}}$ son tales que la función $\Psi(x)$ es de "cuadrado integrable", es decir

$$
\|\Psi(x)\|^{2}=\langle\Psi(x) \mid \Psi(x)\rangle=\sum_{\left\{x_{j}\right\}}\left|\Psi_{x_{j}}\right|^{2}<\infty,
$$

con lo cual $\Psi_{x_{j}}$ será no nulo en un conjunto numerable de puntos. Este espacio de Hilbert es el empleado en la mecánica cuántica polimérica (Ashtekar et al., 2003), la cual es una versión en grados de libertad finitos de la cuantización por lazos. La representación de los generadores del álgebra de Weyl está dada de la forma usual

$$
\widehat{W}^{\prime}(\mu, v) \Psi(x)=e^{\frac{i}{2 \hbar} \mu v} e^{\frac{i}{\hbar} \mu x} \Psi(x+v),
$$


pero ahora $\Psi(x) \in \mathcal{H}^{\prime}$ y la función $\omega_{\mathcal{H}^{\prime}}$ es igual a

$$
\omega_{\mathcal{H}^{\prime}}=\left\langle\delta_{0, x}\left|\widehat{W}^{\prime}(\mu, v)\right| \delta_{0, x}\right\rangle=\int_{\mathbb{R}_{d}} d x_{c} \delta_{0, x} \widehat{W}^{\prime}(\mu, v) \delta_{0, x}=\int_{\mathbb{R}_{d}} d x_{c} \delta_{0, x} e^{\frac{i}{2 \hbar} \mu v} e^{\frac{i}{\hbar} \mu x} \delta_{0, x+v}=e^{\frac{i}{2 \hbar} \mu v} \delta_{0, v}
$$

Podemos ver entones que $\omega_{\mathcal{H}^{\prime}}$ no es una función continua y aunque la representación es irreducible no satisface las condiciones del Teorema de SvN. Esta representación constituye un ejemplo claro en el que los requisitos del Teorema de SvN no son satisfechos y por lo tanto, no será unitariamente equivalente a la representación de Schrödinger. Una implicación inmediata de esto es que no existirá el análogo al operador de traslaciones $\widehat{p}$, haciendo el análisis mucho más complejo (Ashtekar et al. 2003). Por último, en el caso de la Teoría Cuántica de Campos no existe versión del Teorema de Stone-von Neumann. Entre los principales problemas en este caso es que sorprendentemente no existe una única representación de Schrödinger sino una infinidad de ellas algunas de las cuales son explícitamente no unitariamente equivalentes a las otras. Esto plantea el siguiente problema: habiendo diferentes tipos de representaciones de Schrödinger no unitariamente equivalentes entre sí, ¿cuál es la físicamente correcta?. En (Corichi et al., 200200) se muestra que el criterio para "fijar"las representaciones correctas es pedir que el análogo a la función $\omega_{T C C}$ quede invariante ante la acción del grupo de Poincaré. Puede verse que con este criterio, se obtienen infinitas representaciones unitariamente equivalentes entre sí y compatibles con las simetría del grupo de Poincaré. Del mismo modo, esto se usa para construir la representación de Fock, que es el análogo a la representación de Heisenberg en Teoría Cuántica de Campos. Con estos resultados, en (Corichi et al., 2002 0 0) se muestran los requisitos mediante los cuales se pueden relacionar las representaciones à la Fock y à la Schrödinger. Sin embargo, esto es solo para el caso específico de un campo escalar real en espacio-tiempos planos o globalmente hiperbólicos. Para concluir, terminemos mencionando que, en efecto, los fundamentos de la MC distan de ser incólumes. Son muchas las preguntas por responder, por eso acá les dejamos a los lectores algunas que nos parecen llamativas: ¿qué sucede si en lugar de tomar $\mathcal{S}$, formada por funciones lineales de $\mathbb{X}$ y $\mathbb{P}$ para generar $\mathcal{W}$, usamos funciones no lineales?, ¿qué sucede si usamos el álgebra generada por otras variables canónicamente relacionadas con las anteriores pero cuya transformación canónica entre ambas sea no lineal?, ¿qué pasa si trabajamos en el espacio de fase extendido en el que el tiempo y el Hamiltoniano son variables canónicas también?. ¿Cómo emerge el Teorema de SvN en estos casos? Podemos ver que queda mucho por comprender, no solo desde el punto de vista físico sino desde el punto de vista físico-matemático. Sin embargo, como diría M. Planck, "Insight must precede application".

\section{REFERENCIAS}

Achilles, R. and Bonfiglioli, A. (2012). The early proofs of the theorem of Campbell, Baker, Hausdorff, and Dynkin. Archive for history of exact sciences, 66(3):295-358.

Agullo, I. and Corichi, A. (2014). Loop quantum cosmology. In Springer Handbook of Spacetime, pages 809-839. Springer.

Ashtekar, A., Fairhurst, S., and Willis, J. L. (2003). Quantum gravity, shadow states and quantum mechanics. Classical and Quantum Gravity, 20(6):1031.

Bargmann, V. (1961). On a hilbert space of analytic functions and an associated integral transform part $\mathrm{i}$. Communications on pure and applied mathematics, 14(3):187-214.

Bojowald, M. (2008). Loop quantum cosmology. Living Reviews in Relativity, 11(1):4.

Corichi, A., Cortez, J., and Quevedo, H. (2002). Schrödinger representation for a scalar field on curved spacetime. Physical Review D, 66(8):085025.

Corichi, A., Cortez, J., and Quevedo, H. (2003). Note on canonical quantization and unitary equivalence in field theory. Classical and Quantum Gravity, 20(7):L83. 
Corichi, A., Cortez, J., and Quevedo, H. (2004). Schrödinger and fock representation for a field theory on curved spacetime. Annals of Physics, 313(2):446-478.

Dirac, P. A. (1925). The fundamental equations of quantum mechanics. Proc. R. Soc. Lond. A, 109(752):642-653.

Dirac, P. A. M. (1978). Directions in physics. lectures delivered during a visit to australia and new zealand, august/september 1975.

Dirac, P. A. M. (1981). The principles of quantum mechanics. Number 27. Oxford university press.

Dixmier, J. (1977). Enveloping algebras, volume 14. Newnes.

Giulini, D. (2003). That strange procedure called quantisation. Quantum Gravity, pages 103-110.

Haag, R. (2012). Local quantum physics: Fields, particles, algebras. Springer Science \& Business Media.

Hall, B. C. (2013). Quantum theory for mathematicians, volume 267. Springer.

Hall, B. C. (2015). Lie groups, Lie algebras, and representations: an elementary introduction, volume 222. Springer.

Heisenberg, W. (1925). Quantum-theoretical re-interpretation of kinematic and mechanical relations. Z. Phys, 33:879-893.

Isham, C. J. (1993). Canonical quantum gravity and the problem of time. In Integrable systems, quantum groups, and quantum field theories, pages 157-287. Springer.

Kuchar, K. (1993). Canonical quantum gravity. General Relativity and Gravitation 1992, pages 119-50.

Moretti, V. (2013). Spectral theory and quantum mechanics, volume 64. Springer.

Odom, B., Hanneke, D., d' Urso, B., and Gabrielse, G. (2006). New measurement of the electron magnetic moment using a one-electron quantum cyclotron. Physical Review Letters, 97(3):030801.

Reed, M. and Simon, B. (1975). II: Fourier Analysis, Self-Adjointness, volume 2. Elsevier.

Schrödinger, E. (2003). Collected papers on wave mechanics, volume 302. American Mathematical Soc.

Strocchi, F. (2008). An introduction to the mathematical structure of quantum mechanics: a short course for mathematicians, volume 28. World Scientific.

Thiemann, T. (2007). Modern canonical quantum general relativity. Cambridge University Press.

von Neumann, J. (1996). Mathematical foundations of quantum mechanics. Princeton University Press, translation - from german edition.

Weyl, H. (1950). The theory of groups and quantum mechanics. Courier Corporation. 